\title{
A PLATEAU IN THE PLANET POPULATION BELOW TWICE THE SIZE OF EARTH
}

\author{
Erik A. Petigura ${ }^{1}$, Geoffrey W. Marcy ${ }^{1}$, and Andrew W. Howard ${ }^{2}$ \\ ${ }^{1}$ Astronomy Department, University of California, Berkeley, CA 94720, USA; epetigura@ berkeley.edu \\ ${ }^{2}$ Institute for Astronomy, University of Hawaii, 2680 Woodlawn Drive, Honolulu, HI 96822, USA \\ Received 2013 February 9; accepted 2013 April 16; published 2013 May 24
}

\begin{abstract}
We carry out an independent search of Kepler photometry for small transiting planets with sizes 0.5-8.0 times that of Earth and orbital periods between 5 and 50 days, with the goal of measuring the fraction of stars harboring such planets. We use a new transit search algorithm, TERRA, optimized to detect small planets around photometrically quiet stars. We restrict our stellar sample to include the 12,000 stars having the lowest photometric noise in the Kepler survey, thereby maximizing the detectability of Earth-size planets. We report 129 planet candidates having radii less than $6 R_{E}$ found in three years of Kepler photometry (quarters 1-12). Forty-seven of these candidates are not in Batalha et al., which only analyzed photometry from quarters 1-6. We gather Keck HIRES spectra for the majority of these targets leading to precise stellar radii and hence precise planet radii. We make a detailed measurement of the completeness of our planet search. We inject synthetic dimmings from mock transiting planets into the actual Kepler photometry. We then analyze that injected photometry with our TERRA pipeline to assess our detection completeness for planets of different sizes and orbital periods. We compute the occurrence of planets as a function of planet radius and period, correcting for the detection completeness as well as the geometric probability of transit, $R_{\star} / a$. The resulting distribution of planet sizes exhibits a power law rise in occurrence from $5.7 R_{E}$ down to $2 R_{E}$, as found in Howard et al. That rise clearly ends at $2 R_{E}$. The occurrence of planets is consistent with constant from $2 R_{E}$ toward $1 R_{E}$. This unexpected plateau in planet occurrence at $2 R_{E}$ suggests distinct planet formation processes for planets above and below $2 R_{E}$. We find that $15.1_{-2.7}^{+1.8} \%$ of solar type stars-roughly one in six-has a 1-2 $R_{E}$ planet with $P=5-50$ days.
\end{abstract}

Key words: planetary systems - stars: statistics - techniques: photometric

Online-only material: color figures

\section{INTRODUCTION}

The Kepler mission has discovered an extraordinary sample of more than 2300 planets with radii ranging from larger than Jupiter to smaller than Earth (Borucki et al. 2011b; Batalha et al. 2012). Cleanly measuring and debiasing this distribution will be one of Kepler's great legacies. Howard et al. (2012, H12 hereafter) took a key step, showing that the planet radius distribution increases substantially with decreasing planet size down to at least $2 R_{E}$. While the distribution of planets of all periods and radii contains a wealth of information, we choose to focus on the smallest planets. Currently, only Kepler is able to make quantitative statements about the occurrence of planets down to $1 R_{E}$.

The occurrence distributions in $\mathrm{H} 12$ were based on planet candidates ${ }^{3}$ detected in the first four months of Kepler photometry (Borucki et al. 2011b). These planet candidates were detected by a sophisticated pipeline developed by the Kepler team Science Operations Center (Twicken et al. 2010; Jenkins et al. 2010a). ${ }^{4}$ Understanding pipeline completeness, the fraction of planets missed by the pipeline as a function of size and period, is a key component to measuring planet occurrence. Pipeline completeness can be assessed by injecting mock dimmings into photometry and measuring the rate at which injected signals are found. The completeness of the official Kepler pipeline has yet

\footnotetext{
3 The term "planet candidate" is used because a handful of astrophysical phenomena can mimic a transiting planet. However, Morton \& Johnson (2011), Morton (2012), and Fressin et al. (2013) have shown that the false positive rate among Kepler candidates is low, generally between $5 \%$ and $15 \%$. 4 Since H12, Batalha et al. (2012) added many candidates, bringing the number of public KOIs (Kepler objects of interest) to $>2300$. In addition, the Kepler team planet search pipeline has continued to evolve (Smith et al. 2012; Stumpe et al. 2012).
}

to be measured in this manner. This was the key reason why H12 were cautious interpreting planet occurrence under $2 R_{E}$.

In this work, we focus on determining the occurrence of small planets. To maximize our sensitivity to small planets, we restrict our stellar sample to include only the 12,000 stars having the lowest photometric noise in the Kepler survey. We comb through quarters 1-12 (Q1-Q12) — three years of Kepler photometry-with a new algorithm, TERRA, optimized to detect low signal-to-noise $(\mathrm{S} / \mathrm{N})$ transit events. We determine TERRA's sensitivity to planets of different periods and radii by injecting synthetic transits into Kepler photometry and measuring the recovery rate as a function of planet period and radius.

We describe our selection of 12,000 low-noise targets in Section 2. We comb their photometry for exoplanet transits with TERRA, introduced in Section 3. We report candidates found with TERRA (Section 4), which we combine with our measurement of pipeline completeness (Section 5) to produce debiased measurements of planet occurrence (Section 6). We offer some comparisons between TERRA planet candidates and those from Batalha et al. (2012) in Section 7 as well as occurrence measured using both catalogs in Section 8. We offer some interpretations of the constant occurrence rate for planets smaller than $2 R_{E}$ in Section 9.

\section{THE Best12k STELLAR SAMPLE}

We restrict our study to the best 12,000 solar type stars from the perspective of detecting transits by Earth-size planets, hereafter, the "Best12k" sample. For the smallest planets, uncertainty in the occurrence distribution stems largely from pipeline incompleteness due to the low $\mathrm{S} / \mathrm{N}$ of an Earth-size transit. 


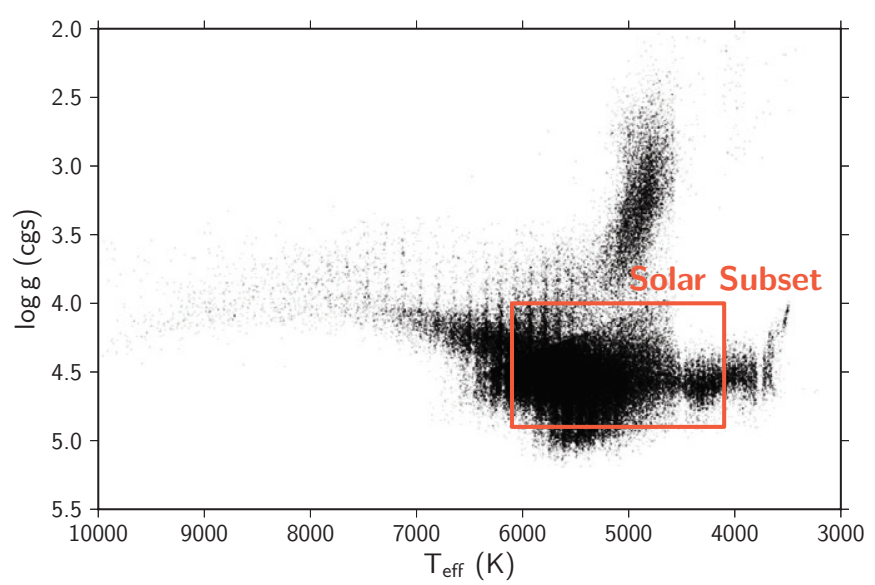

Figure 1. Kepler target stars observed every quarter from Q1-Q9. The rectangle marks the "solar subset" of stars with $T_{\text {eff }}=4100-6100 \mathrm{~K}$ and $\log g=$ 4.0-4.9 (cgs).

(A color version of this figure is available in the online journal.)

Our initial sample begins with the 102,835 stars that were observed during every quarter from Q1-Q9. ${ }^{5}$ From this sample, following H12, we select 73,757 "solar subset" stars that are solar-type $\mathrm{G}$ and $\mathrm{K}$ having $T_{\text {eff }}=4100-6100 \mathrm{~K}$ and $\log g=$ $4.0-4.9$ (cgs). $T_{\text {eff }}$ and $\log g$ values are present in the Kepler Input Catalog (KIC; Brown et al. 2011) which is available online. ${ }^{6}$ Figure 1 shows the KIC-based $T_{\text {eff }}$ and $\log g$ values as well as the solar subset. KIC stellar parameters have large uncertainties: $\sigma(\log g) \sim 0.4$ dex and $\sigma\left(T_{\text {eff }}\right) \sim 200 \mathrm{~K}$ (Brown et al. 2011). As we will discuss in Section 4, we determine stellar parameters for the majority of TERRA planet candidates spectroscopically. For the remaining cases, we use stellar parameters that were determined photometrically, but incorporated a main sequence prior (Batalha et al. 2012). After refining the stellar parameters, we find that 10 of the 129 TERRA planet candidates fall outside of the $T_{\text {eff }}=4100-6100 \mathrm{~K}$ and $\log g=4.0-4.9$ (cgs) solar subset.

From the 73,757 stars that pass our cuts on $\log g$ and $T_{\text {eff }}$, we choose the 12,000 lowest noise stars. Kepler target stars have a wide range of noise properties, and there are several ways of quantifying photometric noise. The Kepler team computes quantities called CDPP3, CDPP6, and CDPP12, which are measures of the photometric scatter in 3, 6, and $12 \mathrm{hr}$ bins (Jenkins et al. 2010a). Since CDPP varies by quarter, we adopt the maximum $6 \mathrm{hr}$ CDPP over Q1-Q9 as our nominal noise metric. We use the maximum noise level (as opposed to median or mean) because a single quarter of noisy photometry can set a high noise floor for planet detection. One may circumvent this problem by removing noisy regions of photometry, which is a planned upgrade to TERRA. Figure 2 shows the distribution of max(CDPP6) among the 73,757 stars considered for our sample.

In choosing our sample, we wanted to include stars amenable to the detection of planets as small as $1 R_{E}$. We picked the 12,000 quietest stars based on preliminary completeness estimates. The noisiest star in the Best12k sample has max(CDPP6) of $79.2 \mathrm{ppm}$. We estimated that the $\sim 100 \mathrm{ppm}$ transit of an Earthsize planet would be detected at $\mathrm{S} / \mathrm{N}_{\mathrm{CDPP}} \sim 1.25 .^{7}$ Given

\footnotetext{
5 We ran TERRA on Q1-Q12 photometry, but we selected the Best12k sample before Q10-Q12 were available.

6 http://archive.stsci.edu/Kepler/kic.html

$7 \mathrm{~S} / \mathrm{N}_{\mathrm{CDPP}}$, the expected $\mathrm{S} / \mathrm{N}$ using the $\max (\mathrm{CDPP} 6)$ metric, is different from the $\mathrm{S} / \mathrm{N}$ introduced in Section 3.2. $\mathrm{S} / \mathrm{N}_{\mathrm{CDPP}}$ is more similar to the $\mathrm{S} / \mathrm{N}$ computed by the Kepler team, which adopts $\mathrm{S} / \mathrm{N}_{\mathrm{CDPP}}>7.1$ as their detection threshold.
}

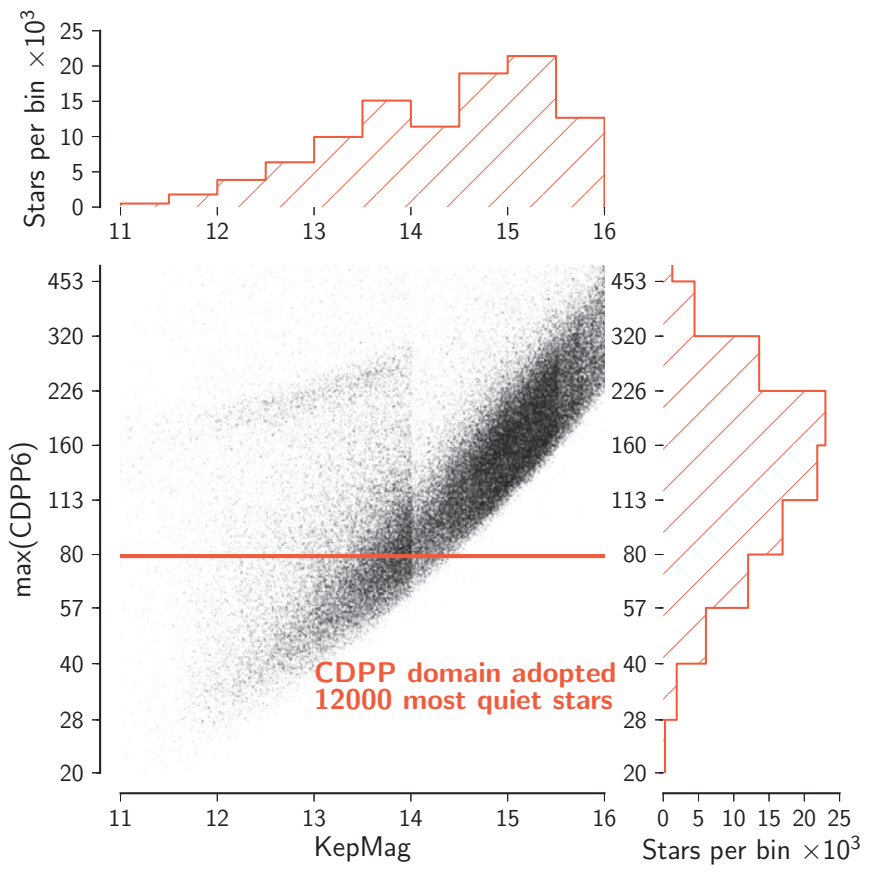

Figure 2. Stellar photometric noise level plotted against Kepler magnitude. Noise level is the maximum value of CDPP6 over Q1-Q9. Of the 73,757 stars that pass our cuts on $T_{\text {eff }}$ and $\log g$, we select the 12,000 most quiet stars. The line shows $\max (\mathrm{CDPP} 6)=79.2 \mathrm{ppm}$, corresponding to the noisiest star in the Best12k sample, well below the median value of $143 \mathrm{ppm}$.

(A color version of this figure is available in the online journal.)

that Q1-Q12 contains roughly 1000 days of photometry, we expected to detect a 5 day planet at $\mathrm{S} / \mathrm{N}_{\mathrm{CDPP}} \sim 1.25 \times$ $\sqrt{1000 / 5} \sim 18$ (a strong detection) and to detect a 50 day planet at $\mathrm{S} / \mathrm{N}_{\mathrm{CDPP}} \sim 1.25 \times \sqrt{1000 / 50} \sim 5.6$ (a marginal detection). In our detailed study of completeness, described in Section 5, we find that TERRA recovers most planets down to $1 R_{E}$ having $P=5-50$ days.

We draw stars from the H12 solar subset for two reasons. First, we may compare our planet occurrence to that of $\mathrm{H} 12$ without the complication of varying occurrence with different stellar types. We recognize that subtle differences may exist between the H12 and Best12k stellar samples. One such difference is that the Best12k is noise-limited, while the H12 sample is magnitude-limited. H12 included bright stars with high photometric variability, which are presumably young and/or active stars. Planet formation efficiency could depend on stellar age. Planets may be less common around older stars that formed before the metallicity of the Galaxy was enriched to current levels. This work assesses planet occurrence for a set of stars that are systematically selected to be 3-10 Gyr old by virtue of their reduced magnetic activity.

The second reason for adopting the $\mathrm{H} 12$ solar subset is a practical consideration of our completeness study. As shown in Section 5, we parameterize pipeline efficiency as a function of $P$ and $R_{P}$. Because M-dwarfs have smaller radii than G-dwarfs, an Earth-size planet dims an M-dwarf more substantially and should be easier for TERRA to detect. Thus, measuring completeness as a function of $P, R_{P}$, and $R_{\star}$ (or perhaps $P$ and $R_{P} / R_{\star}$ ) is appropriate when analyzing stars of significantly different sizes. Such extensions are beyond the scope of this paper, and we consider stars with $R_{\star} \sim R_{\odot}$. 


\section{PLANET SEARCH PIPELINE}

Identifying the smallest transiting planets in Kepler photometry requires a sophisticated automated pipeline. Our pipeline is called "TERRA" and consists of three major components. First, TERRA calibrates photometry in the time domain. Then, TERRA combs the calibrated photometry for periodic, box-shaped signals by evaluating the $\mathrm{S} / \mathrm{N}$ over a finely-spaced grid in transit period $(P)$, epoch $\left(t_{0}\right)$ and duration $(\Delta T)$. Finally, TERRA fits promising signals with a Mandel \& Agol (2002) transit model and rejects signals that are not consistent with an exoplanet transit. We review the calibration component in Section 3.1, but refer the reader to Petigura \& Marcy (2012) for a detailed description. We present, for the first time, the grid-search and light curve fitting components in Sections 3.2 and 3.3.

\subsection{Photometric Calibration}

We briefly review the major time domain components of TERRA; for a more complete description, please refer to Petigura \& Marcy (2012). We begin with Kepler "simple aperture long cadence photometry," which we downloaded from the Mikulski Archive for Space Telescopes. This photometry is the total photoelectrons accumulated within a predefined target aperture over a 29.4 minute interval (Fraquelli \& Thompson 2012). We remove thermal settling events manually and cosmic rays using a median filter. Next, we remove photometric trends longer than 10 days with a high-pass filter. Finally, we identify photometric modes shared by a large ensemble of stars by using a robust principal components analysis. The optimum linear combination of the four most significant modes is removed from each light curve individually.

\subsection{Grid-based Transit Search}

We then search for periodic, box-shaped signals in ensemblecalibrated photometry. Such a search involves evaluating the $\mathrm{S} / \mathrm{N}$ over a finely sampled grid in period $(P)$, epoch $\left(t_{0}\right)$, and duration $(\Delta T)$, i.e.,

$$
\mathrm{S} / \mathrm{N}=\mathrm{S} / \mathrm{N}\left(P, t_{0}, \Delta T\right)
$$

Our approach is similar to the widely-used BLS algorithm of Kovács et al. (2002) as well as to the TPS component of the Kepler pipeline (Jenkins et al. 2010b). BLS, TPS, and TERRA are all variants of a "matched filter" (North 1963). The way in which such an algorithm searches through $P, t_{0}$, and $\Delta T$ is up to the programmer. We choose to search first through $\Delta T$ (outer loop), then $P$, and finally $t_{0}$ (inner loop).

For computational simplicity, we consider transit durations that are integer numbers of long cadence measurements. Since we search for transits with $P=5-50$ days, we try $\Delta T=$ $[3,5,7,10,14,18]$ long cadence measurements, which span the range of expected transit durations, $1.5-8.8 \mathrm{hr}$, for $\mathrm{G}$ and $\mathrm{K}$ dwarf stars.

After choosing $\Delta T$, we compute the mean depth, $\overline{\delta F}\left(t_{i}\right)$, of a putative transit with duration $=\Delta T$ centered at $t_{i}$ for each cadence. $\overline{\delta F}$ is computed via

$$
\overline{\delta F}\left(t_{i}\right)=\sum_{j} F\left(t_{i-j}\right) G_{j}
$$

where $F\left(t_{i}\right)$ is the median-normalized stellar flux at time $t_{i}$ and $G_{j}$ is the $j$ th element of the following kernel

$$
\mathbf{G}=\frac{1}{\Delta T}[\underbrace{\frac{1}{2}, \ldots, \frac{1}{2}}_{\Delta T}, \underbrace{-1, \ldots,-1}_{\Delta T}, \underbrace{\frac{1}{2}, \ldots, \frac{1}{2}}_{\Delta T}] .
$$

As an example, if $\Delta T=3$,

$$
\mathbf{G}=\frac{1}{3}\left[\frac{1}{2}, \frac{1}{2}, \frac{1}{2},-1,-1,-1, \frac{1}{2}, \frac{1}{2}, \frac{1}{2}\right] .
$$

We search over a finely sampled grid of trial periods from 5 to 50 days and epochs ranging from $t_{\text {start }}$ to $t_{\text {start }}+P$, where $t_{\text {start }}$ is the time of the first photometric observation. For a given $\left(P, t_{0}, \Delta T\right)$ there are $N_{T}$ putative transits with depths $\overline{\delta F}_{i}$, for $i=0,1, \ldots, N_{T}-1$. For each $\left(P, t_{0}, \Delta T\right)$ triple, we compute $\mathrm{S} / \mathrm{N}$ from

$$
\mathrm{S} / \mathrm{N}=\frac{\sqrt{N_{T}}}{\sigma} \text { mean }\left(\overline{\delta F}_{i}\right)
$$

where $\sigma$ is a robust estimate (median absolute deviation) of the noise in bins of length $\Delta T$.

For computational efficiency, we employ the "fast folding algorithm" (FFA) of Staelin (1969) as implemented in E. A. Petigura \& G. W. Marcy (2013, in preparation). Let $P_{\text {cad, } 0}$ be a trial period that is an integer number of long cadence measurements, e.g., $P_{\text {cad }, 0}=1000$ implies $P=1000 \times$ 29.4 minutes $=20.43$ days. Let $N_{\text {cad }}=51413$ be the length of the Q1-Q12 time series measured in long cadences. Leveraging the FFA, we compute $\mathrm{S} / \mathrm{N}$ at the following periods:

$$
P_{\text {cad }, i}=P_{\text {cad }, 0}+\frac{i}{M-1} ; i=0,1, \ldots, M-1
$$

where $M=N_{\text {cad }} / P_{\text {cad, } 0}$ rounded up to the nearest power of two. In our search from 5 to 50 days, $P_{\text {cad, } 0}$ ranges from 245 to 2445 , and we evaluate $\mathrm{S} / \mathrm{N}$ at $\sim 10^{5}$ different periods. At each $P_{\text {cad }, i}$ we evaluate $\mathrm{S} / \mathrm{N}$ for $P_{\text {cad, } 0}$ different starting epochs. All told, for each star, we evaluate $\mathrm{S} / \mathrm{N}$ at $\sim 10^{9}$ different combinations of $P, t_{0}$, and $\Delta T$.

Due to runtime and memory constraints, we store only one $\mathrm{S} / \mathrm{N}$ value for each of the trial periods. TERRA stores the maximum $\mathrm{S} / \mathrm{N}$ at that period for all $\Delta T$ and $t_{0}$. We refer to this one-dimensional distribution of $\mathrm{S} / \mathrm{N}$ as the "S/N periodogram," and we show the KIC-3120904 S/N periodogram in Figure 3 as an example. Because we search over many $\Delta T$ and $t_{0}$ at each trial period, fluctuations often give rise to $\mathrm{S} / \mathrm{N} \sim 8$ events and set the detectability floor in the S/N periodogram. For KIC-3120904, a star not listed in the Batalha et al. (2012) planet catalog, we see an $\mathrm{S} / \mathrm{N}$ peak of 16.6 , which rises clearly above stochastic background.

If the maximum $\mathrm{S} / \mathrm{N}$ in the $\mathrm{S} / \mathrm{N}$ periodogram exceeds 12 , we pass that particular $\left(P, t_{0}, \Delta T\right)$ on to the "data validation" (DV) step, described in the following section, for additional vetting. We chose 12 as our $\mathrm{S} / \mathrm{N}$ threshold by trial and error. Note that the median absolute deviation of many samples drawn from a Gaussian distribution is 0.67 times the standard deviation, i.e., $\sigma_{\mathrm{MAD}}=0.67 \sigma_{\mathrm{STD}}$. Therefore, TERRA S $/ \mathrm{N}=12$ corresponds roughly to $\mathrm{S} / \mathrm{N}=8$ in a BLS or TPS search.

Since TERRA only passes the $\left(P, t_{0}, \Delta T\right)$ triple with the highest $\mathrm{S} / \mathrm{N}$ on to DV, TERRA does not detect additional planets with lower $\mathrm{S} / \mathrm{N}$ due to either smaller size or longer 


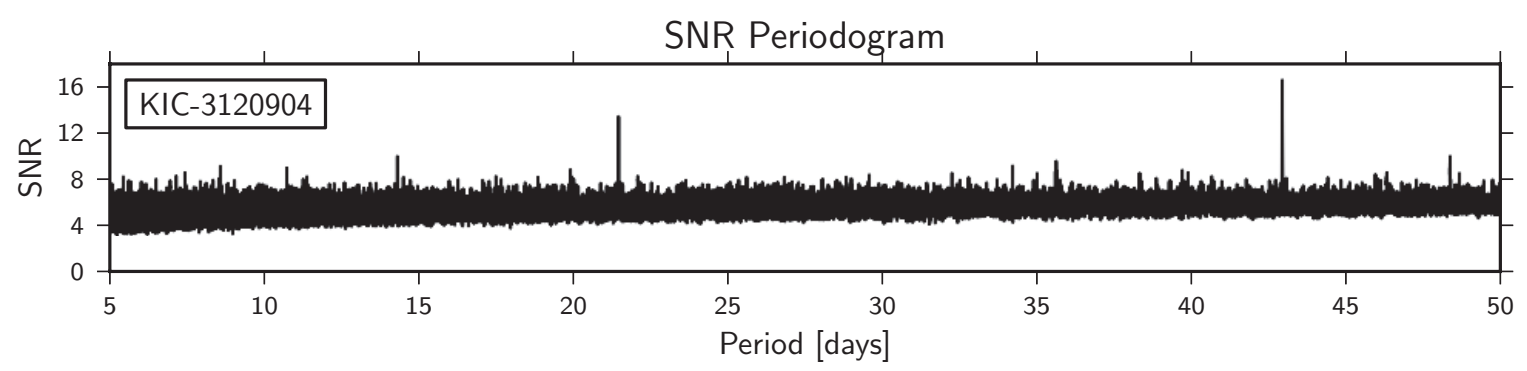

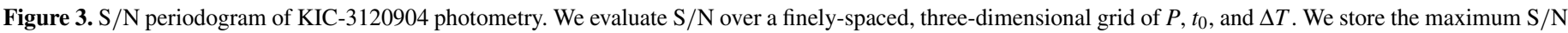

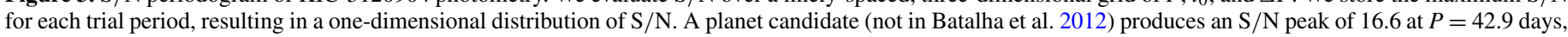
which rises clearly above the detection floor of $\mathrm{S} / \mathrm{N} \sim 8$.

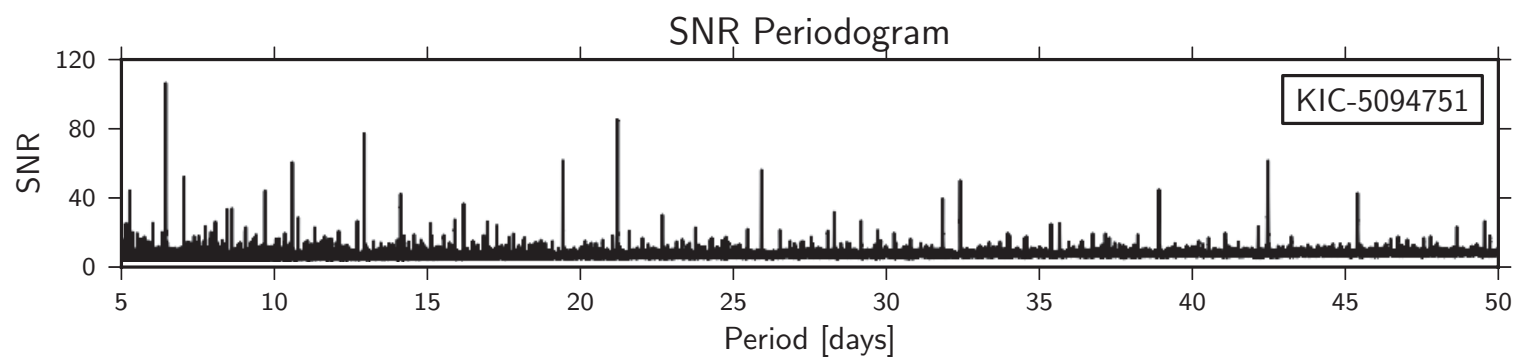

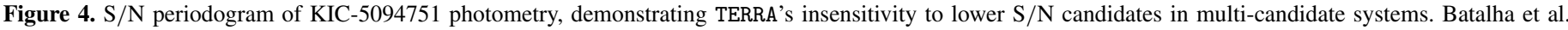

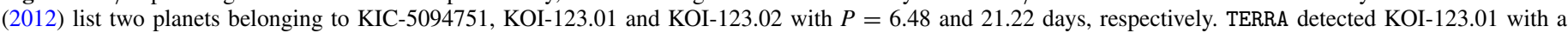

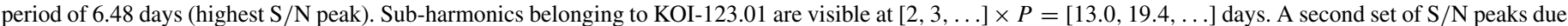

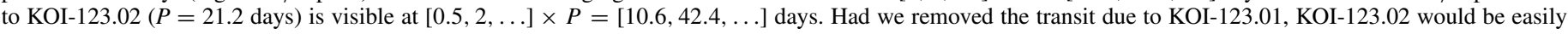
detectible due its high $\mathrm{S} / \mathrm{N}$ of $\sim 80$. TERRA does not yet include multi-candidate logic and is thus blind to lower $\mathrm{S} / \mathrm{N}$ candidates in multi-candidate systems.

orbital period. As an example of TERRA's insensitivity to small candidates in multi-candidate systems, we show the TERRA S/N periodogram for KIC-5094751 in Figure 4. Batalha et al. (2012) list two candidates belonging to KIC-5094751: KOI-123.01 and KOI-123.02 with $P=6.48$ and 21.22 days, respectively. Although the $\mathrm{S} / \mathrm{N}$ periodogram shows two sets of peaks coming from two distinct candidates, TERRA only identifies the first peak. Automated identification of multi-candidate systems is a planned upgrade for TERRA. Another caveat is that TERRA assumes strict periodicity and struggles to detect low $\mathrm{S} / \mathrm{N}$ transits with significant transit timing variations, i.e., variations longer than the transit duration.

\subsection{Data Validation}

If the $\mathrm{S} / \mathrm{N}$ periodogram has a maximum $\mathrm{S} / \mathrm{N}$ peak $>12$, we flag the corresponding $\left(P, t_{0}, \Delta T\right)$ for additional vetting. Following the language of the official Kepler pipeline, we refer to these triples as "threshold crossing events" (TCEs), since they have high photometric $\mathrm{S} / \mathrm{N}$, but are not necessarily consistent with an exoplanet transit. TERRA vets the TCEs in a step called "DV," again following the nomenclature of the official Kepler pipeline. DV, as implemented in the official Kepler pipeline, is described in Jenkins et al. (2010a). We emphasize that TERRA DV does not depend on the DV component of the Kepler team pipeline.

We show the distribution of maximum $\mathrm{S} / \mathrm{N}$ for each Best $12 \mathrm{k}$ star in Figure 5. Among the Best12k stars, 738 have a maximum $\mathrm{S} / \mathrm{N}$ peak exceeding 12 . Adopting $\mathrm{S} / \mathrm{N}=12$ as our threshold balances two competing needs: the desire to recover small planets (low $\mathrm{S} / \mathrm{N}$ ) and the desire to remove as many non-transit events as possible before DV (high $\mathrm{S} / \mathrm{N}$ ). As discussed below, only 129 out of all 738 events with $\mathrm{S} / \mathrm{N}>12$ are consistent with an exoplanet transit, with noise being responsible for the remaining 609. As shown in Figure 5, that number grows rapidly

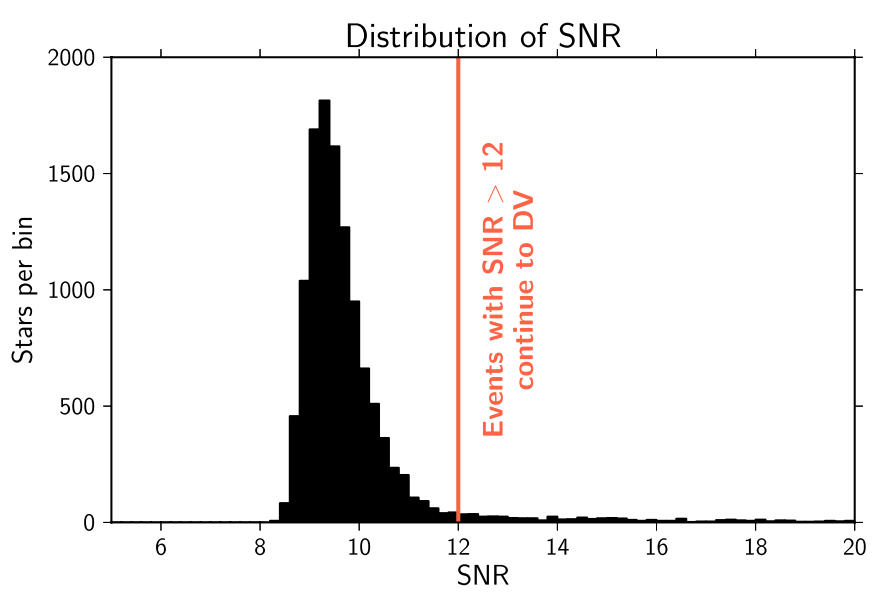

Figure 5. Distribution of the highest $\mathrm{S} / \mathrm{N}$ peak for each star in the Best12k sample. We show $\mathrm{S} / \mathrm{N}=5-20$ to highlight the distribution of low $\mathrm{S} / \mathrm{N}$ events. The 738 stars with $\mathrm{S} / \mathrm{N}>12$ are labeled "threshold crossing events" (TCEs) and are subjected to additional scrutiny in the "data validation" component of TERRA.

(A color version of this figure is available in the online journal.)

as we lower the $\mathrm{S} / \mathrm{N}$ threshold. For example, the number of TCEs grows to 3055 with an $\mathrm{S} / \mathrm{N}$ threshold of 10, dramatically increasing the burden on the DV component.

A substantial number (347) of TCEs are due to harmonics or subharmonics of TCEs outside of the $P=5-50$ day range and are discarded. In order to pass DV, a TCE must also pass a suite of four diagnostic metrics. The metrics are designed to test whether a light curve is consistent with an exoplanet transit. We describe the four metrics in Table 1 along with the criteria the TCE must satisfy in order pass DV. The metrics and cuts were determined by trial and error. We recognize that the TERRA DV metrics and cuts are not optimal and discard a small number of 


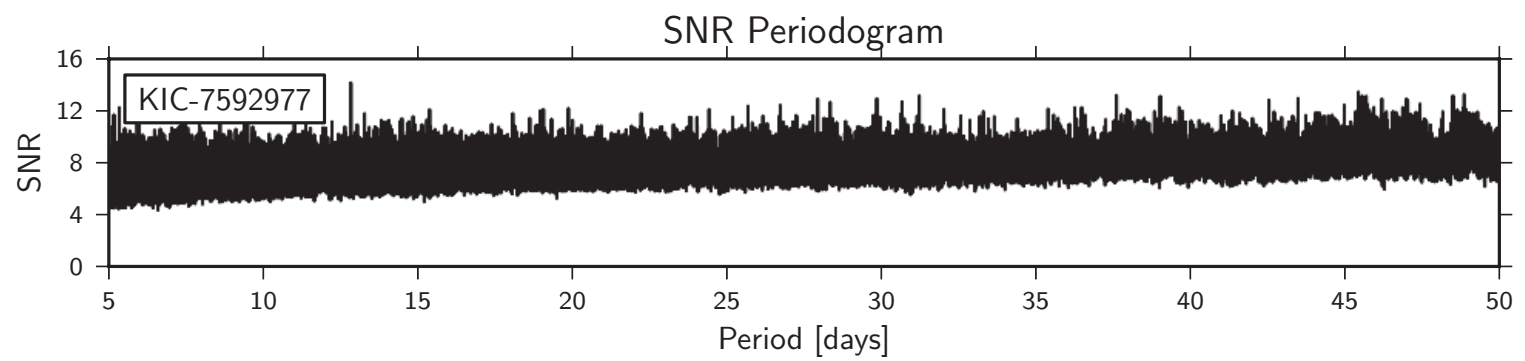

Figure 6. S/N periodogram of KIC-7592977, which passed the automated DV cuts, but was removed manually. KIC-7592977 photometry exhibited short bursts of high photometric scatter, which raised the noise floor to $\mathrm{S} / \mathrm{N} \sim 12$, up from $\mathrm{S} / \mathrm{N} \sim 8$ as in most stars.

Table 1

In Order to Pass the "Data Validation" (DV) Stage, a "Threshold Crossing Event" (TCE) must Pass the Following Suite of Cuts

\begin{tabular}{ll}
\hline \hline Name & \multicolumn{1}{c}{ Description } \\
\hline s2n_out_on_in & $\begin{array}{l}\text { Compelling transits have flat out-of-transit light curves. For a TCE with }\left(P, t_{0}, \Delta T\right) \text {, we remove the transit region from the light } \\
\text { curve and evaluate the } \mathrm{S} / \mathrm{N} \text { of all other }\left(P^{\prime}, t_{0}^{\prime}, \Delta T^{\prime}\right) \text { triples where } P=P^{\prime} \text { and } \Delta T=\Delta T^{\prime} . \mathrm{s} 2 \mathrm{n}_{-} \text {out_on_in is the ratio of the } \\
\text { two highest } \mathrm{S} / \mathrm{N} \text { events. }\end{array}$ \\
med_on_mean & $\begin{array}{l}\text { Since the our definition of } \mathrm{S} / \mathrm{N} \text { (Equation }(5)) \text { depends on the arithmetic mean of individual transit depths, outliers occasionally } \\
\text { produce high } \mathrm{S} / \mathrm{N} \text { TCEs. For each TCE, we compute a robust } \mathrm{S} / \mathrm{N},\end{array}$ \\
$\quad$ med $\mathrm{S} / \mathrm{N}=\frac{\sqrt{N_{T}}}{\sigma}$ median $\left(\overline{\delta F}_{i}\right)$. \\
autor \\
taur
\end{tabular}

compelling exoplanet candidates, as discussed in Section 7.3. However, since we measure TERRA's completeness by injection and recovery of synthetic transits, the sub-optimal nature of our metrics and cuts is incorporated into our completeness corrections.

Our suite of automated cuts removes all but 145 TCEs. We perform a final round of manual vetting and remove 16 additional TCEs, leaving 129 planet candidates. Most TCEs that we remove manually come from stars with highly nonstationary photometric noise properties. Some stars have small regions of photometry that exceed typical noise levels by a factor of three. We show the $\mathrm{S} / \mathrm{N}$ periodogram for one such star, KIC-7592977, in Figure 6. Our definition of S/N (Equation (5)) incorporates a single measure of photometric scatter based on the median absolute deviation, which is insensitive to short bursts of high photometric variability. In such stars, fluctuations readily produce $\mathrm{S} / \mathrm{N} \sim 12$ events and raise the detectability floor to $\mathrm{S} / \mathrm{N} \sim 12$, up from $\mathrm{S} / \mathrm{N} \sim 8$ in most stars. We also visually inspect phase-folded light curves for coherent out-oftransit variability, not caught by our automated cuts, and for evidence of a secondary eclipse.

\section{SMALL PLANETS FOUND BY TERRA}

Out of the 12,000 stars in the Best12k sample, TERRA detected 129 planet candidates achieving $\mathrm{S} / \mathrm{N}>12$ that passed our suite of DV cuts as well as visual inspection. Table 2 in the Appendix lists the 129 planet candidates. We derive planet radii using $R_{P} / R_{\star}$ (from Mandel-Agol model fits) and $R_{\star}$ from spectroscopy (when available) or broadband photometry.

We obtained spectra for 100 of the 129 stars using HIRES (Vogt et al. 1994) at the Keck I telescope with the standard configuration of the California Planet Survey (Marcy et al. 2008). These spectra have resolution of $\sim 50,000$, at an $S / N$ of 45 pixel $^{-1}$ at $5500 \AA$. We determine stellar parameters using a routine called SpecMatch (A. W. Howard et al. 2013, in preparation). In brief, SpecMatch compares a stellar spectrum to a library of $\sim 800$ spectra with $T_{\text {eff }}=3500-7500 \mathrm{~K}$ and $\log g=2.0-5.0$ (determined from LTE spectral modeling). Once the target spectrum and library spectrum are placed on the same wavelength scale, we compute $\chi^{2}$, the sum of the squares of the pixel-by-pixel differences in normalized intensity. The weighted mean of the 10 spectra with the lowest $\chi^{2}$ values is taken as the final value for the effective temperature, stellar surface gravity, and metallicity. We estimate SpecMatchderived stellar radii are uncertain to $10 \% \mathrm{rms}$, based on tests of stars having known radii from high resolution spectroscopy and asteroseismology.

For 27 stars where spectra are not available, we adopt the photometrically-derived stellar parameters of Batalha et al. (2012). These parameters are taken from the KIC (Brown et al. 2011), but then modified so that they lie on the Yonsei-Yale stellar evolution models of Demarque et al. (2004). The resulting stellar radii have uncertainties of $35 \%$ (rms), but can be incorrect by a factor of two or more. As an extreme example, the interpretations of the three planets in the KOI-961 system (Muirhead et al. 2012) changed dramatically when HIRES spectra showed the star to be an M5 dwarf $\left(0.2 R_{\odot}\right.$ as opposed to $0.6 R_{\odot}$ listed in the $\left.\mathrm{KIC}\right)$. We could not obtain spectra for two stars, KIC-7345248 and KIC-8429668, which were not present in Batalha et al. (2012). We determine stellar parameters for these stars by fitting the KIC photometry to Yonsei-Yale stellar models. We adopt $35 \%$ fractional errors on photometricallyderived stellar radii. 


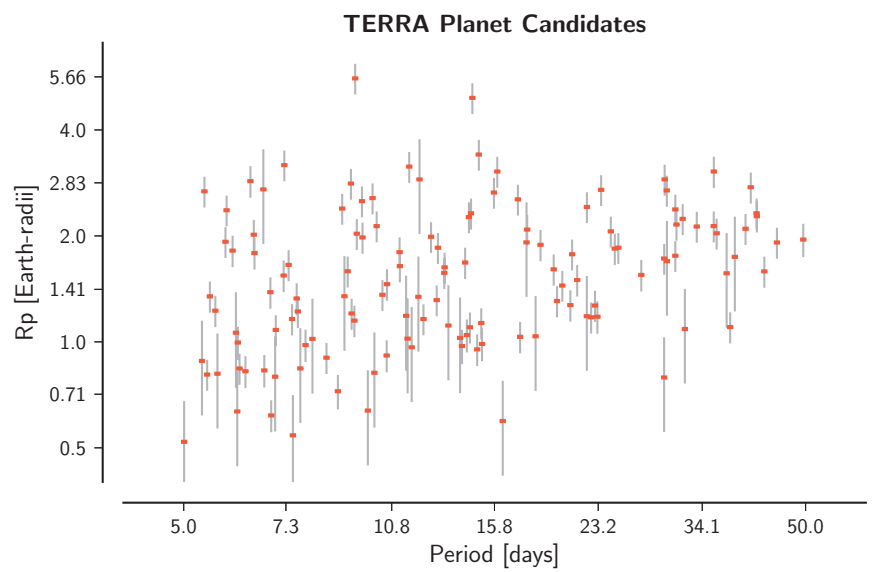

Figure 7. Periods and radii of 129 planet candidates detected by TERRA. Errors on $R_{P}$ are computed via $\sigma\left(R_{P}\right) / R_{P}=$ $\sqrt{\left(\sigma\left(R_{\star}\right) / R_{\star}\right)^{2}+\left(\sigma\left(R_{P} / R_{\star}\right) /\left(R_{P} / R_{\star}\right)\right)^{2}}$, where $R_{P} / R_{\star}$ is the radius ratio. The error in $R_{P}$ stems largely from the uncertainty in stellar radii. We adopt $\sigma\left(R_{\star}\right) / R_{\star}=10 \%$ for the 100 stars with spectroscopically determined $R_{\star}$ and $\sigma\left(R_{\star}\right) / R_{\star}=35 \%$ for the remaining stars with $R_{\star}$ determined from photometry. Using MCMC, we find the uncertainty in $R_{P} / R_{\star}$ is generally $<5 \%$ and thus a minor component of the overall error budget.

(A color version of this figure is available in the online journal.)

Once we determine $P$ and $t_{0}$, we fit a Mandel \& Agol (2002) model to the phase-folded photometry. Such a model has three free parameters: $R_{P} / R_{\star}$, the planet to stellar radius ratio; $\tau$, the time for the planet to travel a distance $R_{\star}$ during transit; and $b$, the impact parameter. In this work, $R_{P} / R_{\star}$ is the parameter of interest. However, $b$ and $R_{P} / R_{\star}$ are covariant, i.e., a transit with $b$ approaching unity only traverses the limb of the star, and thus produces a shallower transit depth. In order to account for this covariance, best fit parameters were computed via Markov Chain Monte Carlo (MCMC). We find that the fractional uncertainty on $R_{P} / R_{\star}, \sigma\left(R_{P} / R_{\star}\right) /\left(R_{P} / R_{\star}\right)$ can be as high as $10 \%$, but is generally less than $5 \%$. Therefore, the error on $R_{P}$ due to covariance with $b$ is secondary to the uncertainty on $R_{\star}$.

We show the distribution of TERRA candidates in Figure 7 over the two-dimensional domain of planet radius and orbital period. Our 129 candidates range in size from $6.83 R_{E}$ to $0.48 R_{E}$ (smaller than Mars). The median TERRA candidate size is 1.58 $R_{E}$. In Figure 8, we show the substantial overlap between the TERRA planet sample and those produced by the Kepler team. TERRA recovers 82 candidates listed in Batalha et al. (2012). We discuss the significant overlap between the two works in detail in Section 7. As of 2012 August 8, 10 of our TERRA candidates were listed as false positives in an internal database of Kepler planet candidates maintained by Jason Rowe (Jason Rowe 2012, private communication) and are shown as blue crosses in Figure 8. We do not include these 10 candidates in our subsequent calculation of occurrence. Table 2 lists the KIC identifier, best fit transit parameters, stellar parameters, planet radius, and Kepler team false positive designation of all 129 candidates revealed by the TERRA algorithm. The best fit transit parameters include orbital period, $P$; time of transit center, $t_{0}$; planet to star radius ratio, $R_{P} / R_{\star}$; time for planet to cross $R_{\star}$ during transit, $\tau$; and impact parameter, $b$. We list the following stellar properties: effective temperature, $T_{\text {eff }}$; surface gravity, $\log g$; and stellar radius, $R_{\star}$.

\section{COMPLETENESS OF PLANET CATALOG}

When measuring the distribution of planets as a function of $P$ and $R_{P}$, understanding the number of missed planets is as important as finding planets themselves. H12 accounted for completeness in a rough sense based on $\mathrm{S} / \mathrm{N}$ considerations. For each star in their sample, they estimated the $\mathrm{S} / \mathrm{N}$ over a range of $P$ and $R_{P}$ using CDPP as an estimate of the photometric noise on transit-length timescales. $\mathrm{H} 12$ chose to accept only planets with $\mathrm{S} / \mathrm{N}>10$ in a single quarter of photometry for stars brighter than $K p=15$. This metric used CDPP and was a reasonable pass on the data, particularly when the pipeline completeness was unknown. Determining expected $\mathrm{S} / \mathrm{N}$ from CDPP does not incorporate the real noise characteristics of the photometry, but instead approximates noise on transit timescales as stationary (CDPP assumed to be constant over a quarter) and Gaussian distributed. Moreover, identifying small transiting planets with transit depths comparable to the noise requires a complex, multistage pipeline. Even if the integrated $\mathrm{S} / \mathrm{N}$ is above some nominal threshold, the possibility of missed planets remains a concern.

We characterize the completeness of our pipeline by performing an extensive suite of injection and recovery experiments. We inject mock transits into raw photometry, run this photometry though the same pipeline used to detect planets, and measure the recovery rate. This simple, albeit brute force, technique captures the idiosyncrasies of the TERRA pipeline that are missed by simple $\mathrm{S} / \mathrm{N}$ considerations.

We perform 10,000 injection and recovery experiments using the following steps:

1. We select a star randomly from the Best $12 \mathrm{k}$ sample.

2. We draw $\left(P, R_{P}\right)$ randomly from log-uniform distributions over 5-50 days and 0.5-16.0 $R_{E}$.

3. We draw impact parameter and orbital phase randomly from uniform distributions ranging from 0 to 1 .

4. We generate a Mandel \& Agol (2002) model.

5. We inject it into the "simple aperture photometry" of the selected star.

We then run the calibration, grid-based search, and DV components of TERRA (Sections 3.1-3.3) on this photometry and calculate the planet recovery rate. We do not, however, perform the visual inspection described in Section 3.3. An injected transit is considered recovered if the following two criteria are met: (1) the highest $\mathrm{S} / \mathrm{N}$ peak passes all $\mathrm{DV}$ cuts and (2) the output period and epoch are consistent with the injected period and epoch to within 0.01 and 0.1 days, respectively.

Figure 9 shows the distribution of recovered simulations as a function of period and radius. Nearly all simulated planets with $R_{P}>1.4 R_{E}$ are recovered, compared to almost none with $R_{P}$ $<0.7 R_{E}$. Pipeline completeness is determined in small bins in $\left(P, R_{P}\right)$-space by dividing the number of successfully recovered transits by the total number of injected transits on a bin-by-bin basis. This ratio is TERRA's recovery rate of putative planets within the Best12k sample. Thus, our quoted completeness estimates only pertain to the low photometric noise Best $12 \mathrm{k}$ sample. Had we selected an even more rarified sample, e.g., the "Best6k," the region of high completeness would extend down toward smaller planets.

\section{OCCURRENCE OF SMALL PLANETS}

Following H12, we define planet occurrence, $f$, as the fraction of a defined population of stars having planets within a domain of planet radius and period, including all orbital inclinations. TERRA, however, is only sensitive to one candidate (highest $\mathrm{S} / \mathrm{N}$ ) per system, so we report occurrence as the fraction of stars 


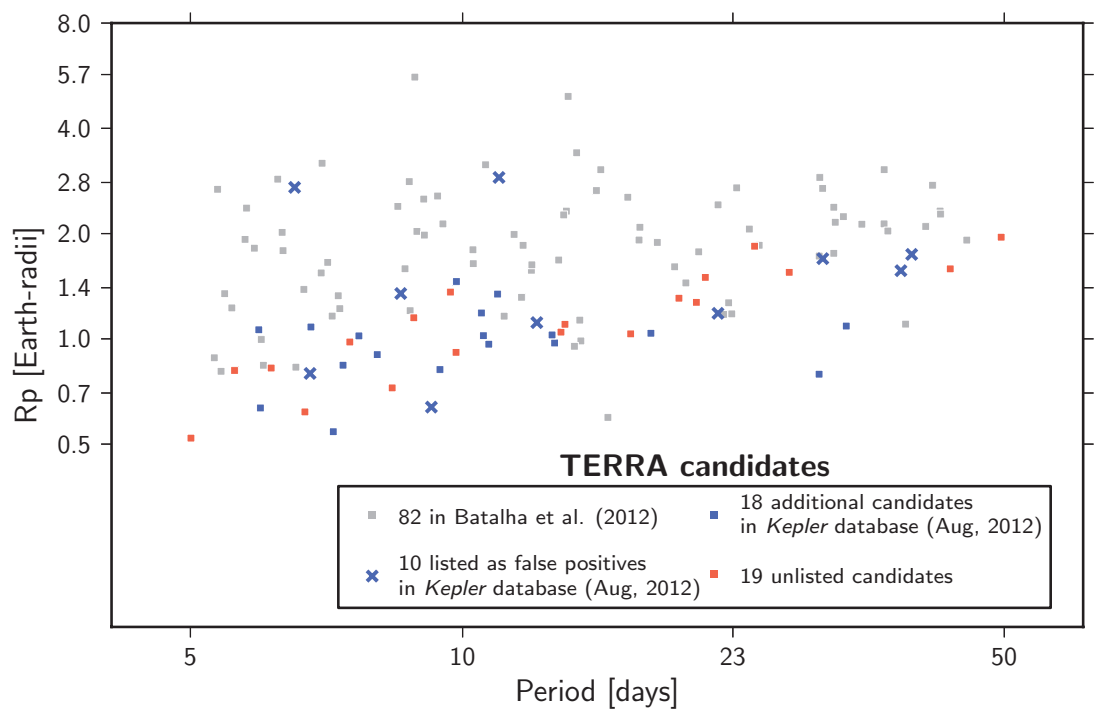

Figure 8. Periods and radii of all 129 TERRA planet candidates. The gray points show candidates that were listed in Batalha et al. (2012). The blue crosses represent candidates deemed false positives by the Kepler team as of 2012 August 8 (Jason Rowe 2012, private communication). These false positives are removed from our sample prior to computing occurrence. Eighteen additional candidates were listed in the same Kepler team database. Red points show 19 unlisted TERRA candidates.
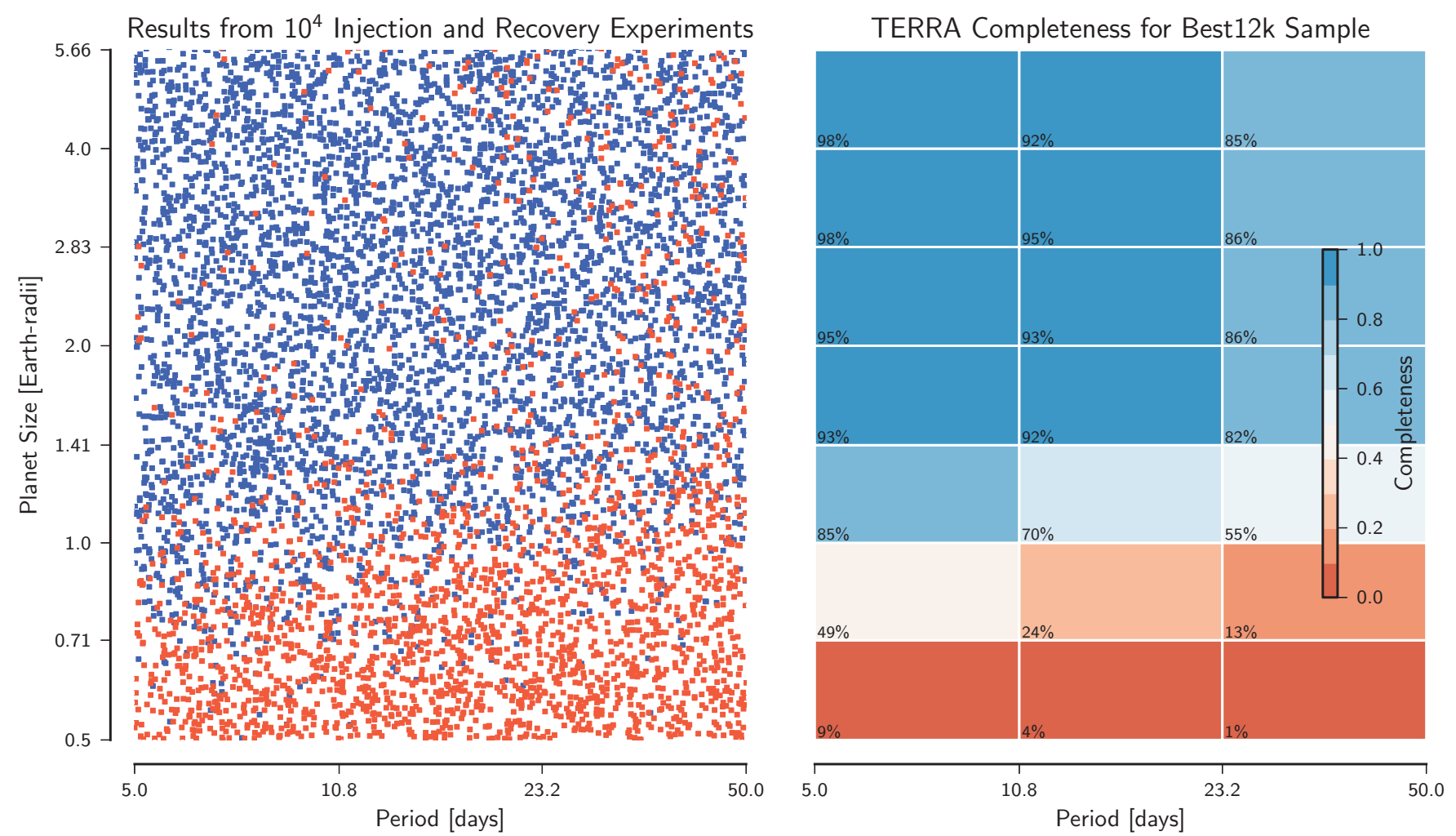

Figure 9. Results from the injection and recovery of 10,000 synthetic transit signals into actual photometry of randomly selected stars from our Best12k stellar sample. Each point represents the planet radius and orbital period of a mock transiting planet. The blue points represent signals that passed the DV post-analysis and where TERRA recovers the correct period and epoch. Signals that did not pass DV and/or were not successfully recovered are shown as red points. Pipeline completeness is simply the number of blue points divided by the total number of points in each bin. The figure shows that for planet sizes above $1.0 R_{E}$, our pipeline discovers over $50 \%$ of the injected planets, and presumably accomplishes a similar success rate for actual transiting planets. The completeness for planets larger than $1 R_{E}$ is thus high enough to compute planet occurrence for such small planets, with only moderate completeness corrections needed (less than a factor of two). Note that we are measuring the recovery rate of putative planets in the Best12k sample with TERRA. Had we selected a lower noise stellar sample, for example the "Best6k," the region of high completeness would extend to even small radii.

with one or more planets with $P=5-50$ days. Our occurrence measurements apply to the Best12k sample of low-noise, solartype stars described in Section 2.

In computing planet occurrence in the Best12k sample, we follow the prescription in $\mathrm{H} 12$ with minor modifications.
Notably, we have accurate measures of detection completeness described in the previous section. In contrast, H12 estimated completeness based on the presumed $\mathrm{S} / \mathrm{N}$ of the transit signal, suffering both from approximate characterization of photometric noise using CDPP and from poor knowledge of 


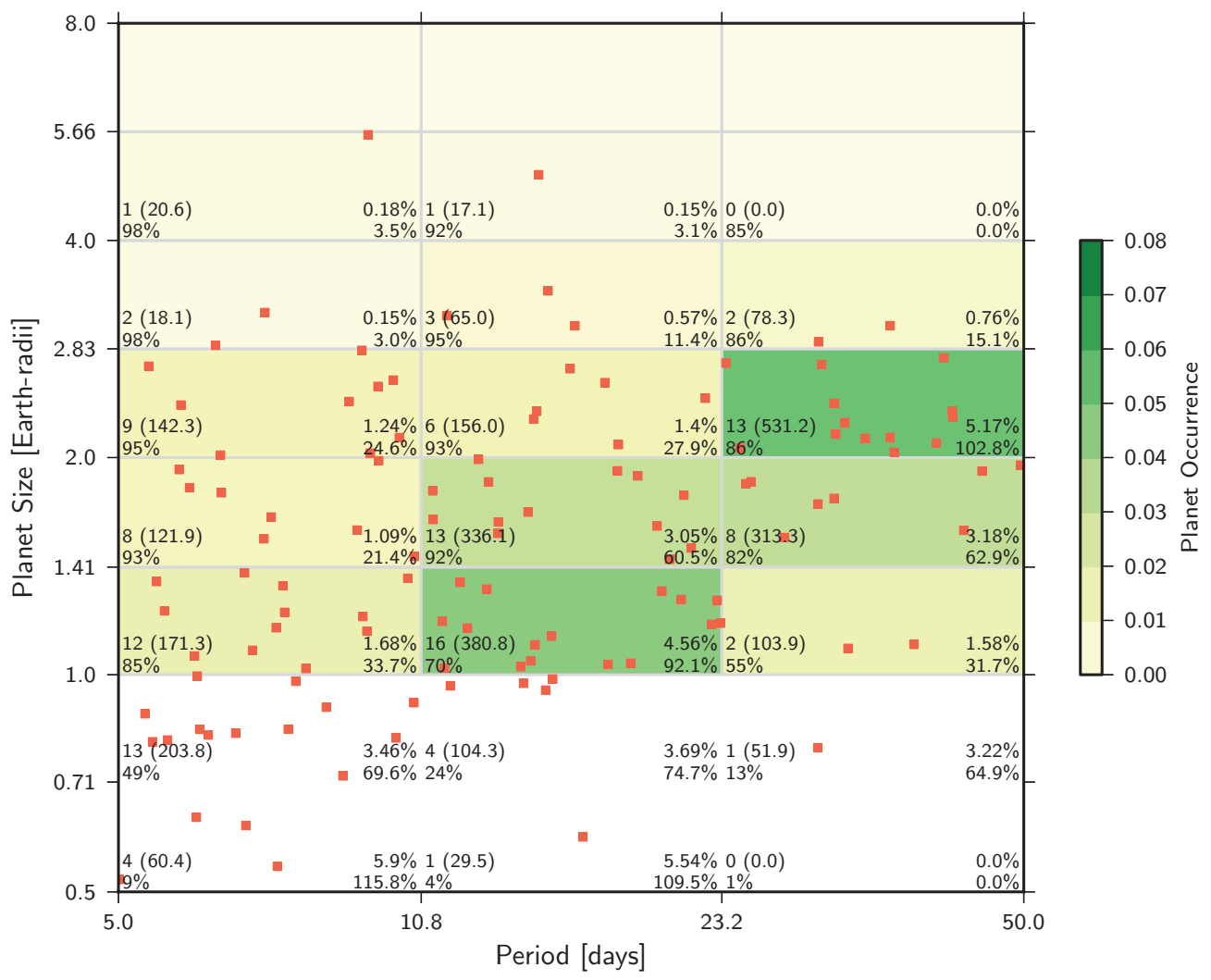

Figure 10. Planet occurrence as a function of orbital period and planet radius for $P=5-50$ days and $R_{P}=0.5-8 R_{E}$. TERRA planet candidates are shown as red points. Cell occurrence, $f_{\text {cell }}$, is given by the color scale. We quote the following information for each cell: Top left: number of planets (number of augmented planets); lower left: completeness; top right: fractional planet occurrence, $f_{\text {cell }}$; bottom right: normalized planet occurrence, $d^{2} f_{\text {cell }} / d \log P / d \log R_{P}$. We do not color cells where the completeness is less than $50 \%$ (i.e., the completeness correction is larger than a factor of two).

the efficiency of the planet-finding algorithm for all periods and sizes.

For each $P-R_{P}$ bin, we count the number of planet candidates, $n_{\mathrm{pl}, \mathrm{cell}}$. Each planet that transits represents many that do not transit given the orientation of their orbital planes with respect to Kepler's line of sight. Assuming random orbital alignment, each observed planet represents $a / R_{\star}$ total planets when nontransiting geometries are considered. For each cell, we compute the number of augmented planets, $n_{\mathrm{pl} \text {,aug,cell }}=\sum_{i} a_{i} / R_{\star, i}$, which accounts for planets with non-transiting geometries. We then use Kepler's third law together with $P$ and $M_{\star}$ to compute $a / R_{\star}$ assuming a circular orbit. ${ }^{8}$

To compute occurrence, we divide the number of stars with planets in a particular cell by the number of stars amenable to the detection of a planet in a given cell, $n_{\star, \text { amen }}$. This number is just $N_{\star}=12,000$ times the completeness, computed in our Monte Carlo study. The debiased fraction of stars with planets per $P-R_{P}$ bin, $f_{\text {cell }}$, is given by $f_{\text {cell }}=n_{\text {pl,aug,cell }} / n_{\star \text {,amen }}$. We show $f_{\text {cell }}$ on the $P-R_{P}$ plane in Figure 10 as a color scale. We also compute $d^{2} f_{\text {cell }} / d \log P / d \log R_{P}$, i.e., planet occurrence divided by the logarithmic area of each cell, which is a measure of occurrence which does not depend on bin size. We annotate each $P-R_{P}$ bin of Figure 10 with the corresponding value of $n_{\mathrm{pl} \text {,cell }}, n_{\mathrm{pl} \text {, aug,cell }}, f_{\text {cell }}$, and $d^{2} f_{\text {cell }} / d \log P / d \log R_{P}$.

Due to the small number of planets in each cell, errors due to counting statistics alone are significant. We compute Poisson errors on $n_{\mathrm{pl} \text {,cell }}$ for each cell. Errors on $n_{\mathrm{pl} \text {,aug,cell }}, f_{\text {cell }}$, and $d^{2} f_{\text {cell }} / d \log P / d \log R_{P}$ include only the Poisson errors from

\footnotetext{
$8 \mathrm{H} 12$ determined $a / R_{\star}$ directly from light curve fits, but found little change
} when computing occurrence from $a / R_{\star}$ using Kepler's third law. $n_{\mathrm{pl}, \mathrm{cell}}$. There is also shot noise associated with the Monte Carlo completeness correction due to the finite number of simulated planets in each $P-R_{P}$ cell, but such errors are small compared to errors on $n_{\mathrm{pl} \text {,cell }}$. The orbital alignment correction, $a / R_{\star}$, is also uncertain due to imperfect knowledge of stellar radii and orbital separations. We do not include such errors in our occurrence estimates.

Of particular interest is the distribution of planet occurrence with $R_{P}$ for all periods. We marginalize over $P$ by summing occurrence over all period bins from 5 to 50 days. The distribution of radii shown in Figure 11 shows a rapid rise in occurrence from 8.0 to $2.8 R_{E}$. H12 also observed a rising occurrence of planets down to $2.0 R_{E}$, which they modeled as a power law. Planet occurrence is consistent with a flat distribution from 2.8 to $1.0 R_{E}$, ruling out a continuation of a power law increase in occurrence for planets smaller than $2.0 R_{E}$. We find $15.1_{-2.7}^{+1.8} \%$ of Sun-like stars harbor a 1.0-2.0 $R_{E}$ planet with $P=5-50$ days. Including larger planets, we find that $24.8_{-3.4}^{+2.1} \%$ of stars harbor a planet larger than Earth with $P=5-50$ days. Occurrence values assuming a $100 \%$ efficient pipeline are shown as gray bars in Figure 11. The red bars show the magnitude of our completeness correction. Even though TERRA detects many planets smaller than $1.0 R_{E}$, we do not report occurrence for planets smaller than Earth since pipeline completeness drops abruptly below $50 \%$.

We show planet occurrence as a function of orbital period in Figure 12. In computing this second marginal distribution, we include radii larger than $1 R_{E}$ so that corrections due to incompleteness are small. Again, as in Figure 11, gray bars represent uncorrected occurrence values while red bars show our correction to account for planets that TERRA missed. Planet 
occurrence rises as orbital period increases from 5.0 to 10.8 days. Above 10.8 days, planet occurrence is nearly constant per logarithmic period bin with a slight indication of a continued rise. This leveling off of the distribution was noted by $\mathrm{H} 12$, who considered $R_{P}>2.0 R_{E}$. We fit the distribution of orbital periods for $R_{P}>1.0 R_{E}$ with two power laws of the form

$$
\frac{d f}{d \log P}=k_{P} P^{\alpha},
$$

where $\alpha$ and $k_{P}$ are free parameters. We find best fit values of $k_{P}=0.185_{-0.035}^{+0.043}, \alpha=0.16 \pm 0.07$ for $P=5-10.8$ days and $k_{P}=8.4_{-0.8}^{+0.9} \times 10^{-3}, \alpha=1.35 \pm 0.05$ for $P=10.8-50$ days. We note that $k_{P}$ and $\alpha$ are strongly covariant. Extrapolating the latter fit speculatively to $P>50$ days, we find $41.7_{-5.9}^{+6.8} \%$ of Sun-like stars host a planet $1 R_{E}$ or larger with $P=50-500$ days.

\section{COMPARISON OF TERRA AND BATALHA ET AL. (2012) PLANET CATALOGS}

Here, we compare our candidates to those of Batalha et al. (2012). Candidates were deemed in common if their periods agree to within 0.01 days. We list the union of the TERRA and Batalha et al. (2012) catalogs in Table 3 in the Appendix. Eightytwo candidates appear in both catalogs (Section 7.1), 47 appear in this work only (Section 7.2), and 33 appear in Batalha et al. (2012) only (Section 7.3). We discuss the significant overlap between the two catalogs and explain why some candidates were detected by one pipeline but not the other.

\subsection{Candidates in Common}

Eighty-two of our candidates appear in the Batalha et al. (2012) catalog. We show these candidates in $P-R_{P}$ space in Figure 8 as gray points. TERRA detected no new candidates with $R_{P}>2 R_{E}$. This agreement in detected planets having $R_{P}>2 R_{E}$ demonstrates high completeness for such planets in both pipelines for this sample of quiet stars. This is not very surprising since candidates with $R_{P}>2 R_{E}$ have high $\mathrm{S} / \mathrm{N}$, e.g., min, median, and $\max \mathrm{S} / \mathrm{N}=19.3,71.5$, and 435 respectively.

Radii for the 82 planets in common were fairly consistent between Batalha et al. (2012) and this work. The two exceptions were KIC-8242434 and KIC-8631504. Using SpecMatch, we find stellar radii of 0.68 and $0.72 R_{\odot}$, respectively, down from 1.86 and $1.80 R_{\odot}$ in Batalha et al. (2012). The revised planet radii are smaller by over a factor of two. Radii for the other planets in common were consistent to $\sim 20 \%$.

\subsection{TERRA Candidates Not in Batalha et al. (2012) Catalog}

TERRA revealed 47 planet candidates that did not appear in Batalha et al. (2012). Such candidates are colored blue and red in Figure 8. Many of these new detections likely stem from the fact that we use twice the photometry that was available to Batalha et al. (2012). To get a sense of how additional photometry improves the planet yield of the Kepler pipeline beyond Batalha et al. (2012), we compared the TERRA candidates to the Kepler team KOI list dated 2012 August 8 (Jason Rowe 2012, private communication). The 28 candidates in common between the 2012 August 8 Kepler team sample and this work are colored blue in Figure 8 . Of these 28 candidates, 10 are listed as false positives and denoted as crosses in Figure 8.

We announce 37 new planet candidates with respect to Batalha et al. (2012) that were not listed as false positives in the
Kepler team sample. These 37 candidates, all with $R_{P} \lesssim 2 R_{E}$, are a subset of those listed in Table 2 . As a convenience, we show this subset in Table 4 in the Appendix. We remind the reader that all photometry used in this work is publicly available. We hope that interested readers will fold the photometry on the ephemeris in Table 2 and assess critically whether a planet interpretation is correct. As a quick reference, we have included plots of the transits of the 37 new candidates from Table 4 in Figures 17 and 18 in the Appendix. We do not claim that our additional candidates bring pipeline completeness to unity for planets with $R_{P} \lesssim 2 R_{E}$. As shown in Section 5, our planet sample suffers from significant incompleteness in the same $P-R_{P}$ space where most of the new candidates emerged.

\subsection{Batalha et al. (2012) Candidates Not in TERRA Catalog}

There are 33 planet candidates in the Batalha et al. (2012) catalog from Best12k stars that TERRA missed. Of these, 28 are multi-candidate systems where one component was identified by TERRA. TERRA is currently insensitive to multiple planet systems (as described in Section 3.2). TERRA missed the remaining five (Batalha et al. 2012) candidates for the following reasons:

1. 2581.01. A bug in the pipeline prevented successful photometric calibration (Section 3.1). This bug affected 19 out of 12,000 stars in the Best12k sample.

2. $70.01,111.01,119.01$. Failed one of the automated DV cuts (taur, med_on_mean, and taur, respectively). We examined these three light curves in the fashion described in Section 3.3, and we determined these light curves were consistent with an exoplanet transit. The fact that DV is discarding compelling transit signals decreases TERRA's overall completeness. Computing DV metrics and choosing the optimum cuts is an art. There is room for improvement here.

3. KOI-1151.01. Period misidentified in Batalha et al. (2012). In Batalha et al. (2012) KOI-1151.01 is listed with a $P=$ 5.22 days. TERRA found a candidate with $P=10.43$ days. Figure 13 shows phase-folded photometry with the TERRA ephemeris. A period of 5.22 days would imply dimmings in regions where the light curve is flat.

We plot the 33 total candidates listed in Batalha et al. (2012), but not found by TERRA in Figure 14. We highlight the five missed candidates that cannot be explained by the fact that they are a lower $\mathrm{S} / \mathrm{N}$ candidate in a multi-candidate system. TERRA is blind to planets in systems with another planet with higher $\mathrm{S} / \mathrm{N}$. Figure 14 shows that most of these missed planets occur at $R_{P}<1.4 R_{E}$.

\section{OCCURRENCE WITH PLANET MULTIPLICITY INCLUDED}

While the TERRA planet occurrence measurement benefits from well-characterized completeness, it does not include the contribution of multis to overall planet occurrence. As discussed in Sections 3.2 and 7.3, TERRA only detects the highest S/N candidate for a given star. Here, we present planet occurrence including multis from Batalha et al. (2012). Thus, the occurrence within a bin, $f$, in this section should be interpreted as the average number of planets per star with $P=5-50$ days. The additional planets from Batalha et al. (2012) raise the occurrence values somewhat over those of the previous section. However, the rise and plateau structure remain the same.

We compute $f_{\text {cell }}$ from the 32 candidates present in Batalha et al. (2012), but not found by TERRA (mislabeled KOI-1151.01 


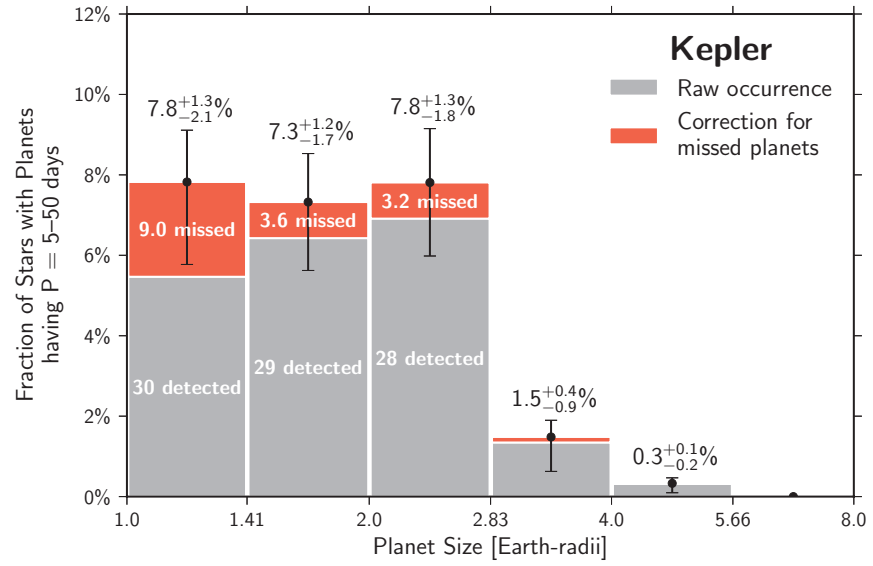

Figure 11. Distribution of planet occurrence for $R_{P}$ ranging from 1.0 to $8.0 R_{E}$. We quote the fraction of Sun-like stars harboring a planet with $P=5-50$ days for each $R_{P}$ bin. We observe a rapid rise in planet occurrence from 8.0 down to $2.8 R_{E}$, as seen in H12. Below $2.8 R_{E}$, the occurrence distribution is consistent with flat. This result rules out a power law increase in planet occurrence toward smaller radii. Adding up the two smallest radius bins, we find $15.1_{-2.7}^{+1.8} \%$ of Sunlike stars harbor a 1.0-2.0 $R_{E}$ planet within $\sim 0.25$ AU. To compute occurrence as a function of $R_{P}$, we simply sum occurrence rates for all period bins shown in Figure 10. Errors due to counting statistics are computed by adding errors from each of the three period bins in quadrature. The gray portion of the histogram shows occurrence values before correcting for missed planets due to pipeline incompleteness. Our correction to account for missed planets is shown in red, and is determined by the injection and recovery of synthetic transits described in Section 5. We do not show occurrence values where the completeness is $<50 \%$.

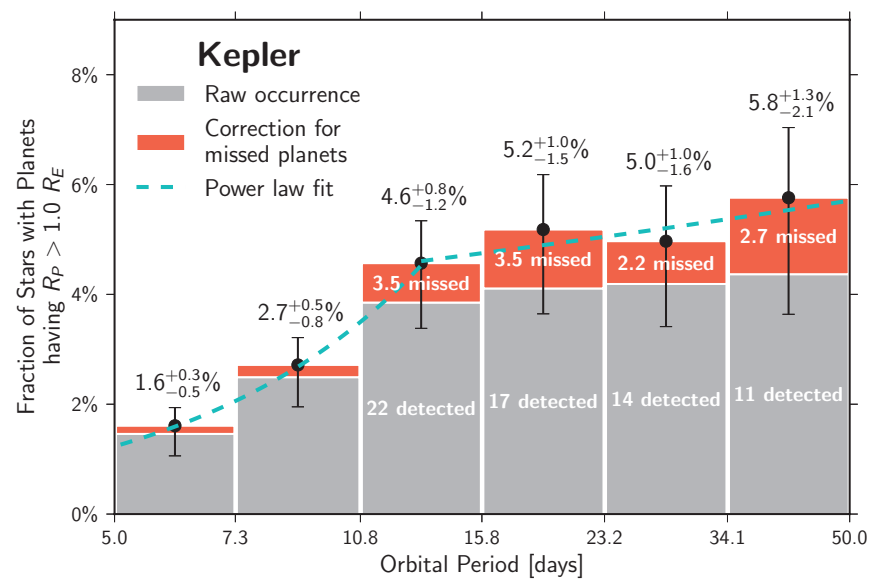

Figure 12. Distribution of planet occurrence for different orbital periods ranging from 5 to 50 days. We quote the fraction of Sun-like stars with a planet Earth-size or larger as a function of orbital period. We observe a gradual rise in occurrence from 5.0 to $10.8 R_{E}$ followed by a leveling off for longer orbital periods. H12 observed a similar leveling off in their analysis which included planets larger than $2 R_{E}$. We fit the domains above and below $10.8 R_{E}$ separately with power laws, $d f / d \log P=k_{P} P^{\alpha}$. We find best fit values of $k_{P}=0.185_{-0.035}^{+0.043}, \alpha=$ $0.16 \pm 0.07$ for $P=5-10.8$ days and $k_{P}=8.4_{-0.8}^{+0.9} \times 10^{-3}, \alpha=1.35 \pm 0.05$ for $P=10.8-50$ days. Speculatively, we extrapolate the latter power law fit another decade in period and estimate $41.7_{-5.9}^{+6.8} \%$ of Sun-like stars harbor a planet Earth-size or larger with $P=50-500$ days. As in Figure 11, the gray portion of the histogram shows uncorrected occurrence while the red region shows our correction for pipeline incompleteness. Note that the number of detected planets decreases as $P$ increases from 10.8 to 50 days, while occurrence remains nearly constant. At longer periods, the geometric transit probability is lower, and each detected planet counts more toward $d f / d \log P$.

was not included). For clarity, we refer to this separate occurrence calculation as $f_{\text {cell,Batalha. }}$ Because the completeness of the Kepler pipeline is unknown, we apply no completeness correction. This assumption of $100 \%$ completeness is certainly an overestimate, but we believe that the sensitivity of the Kepler

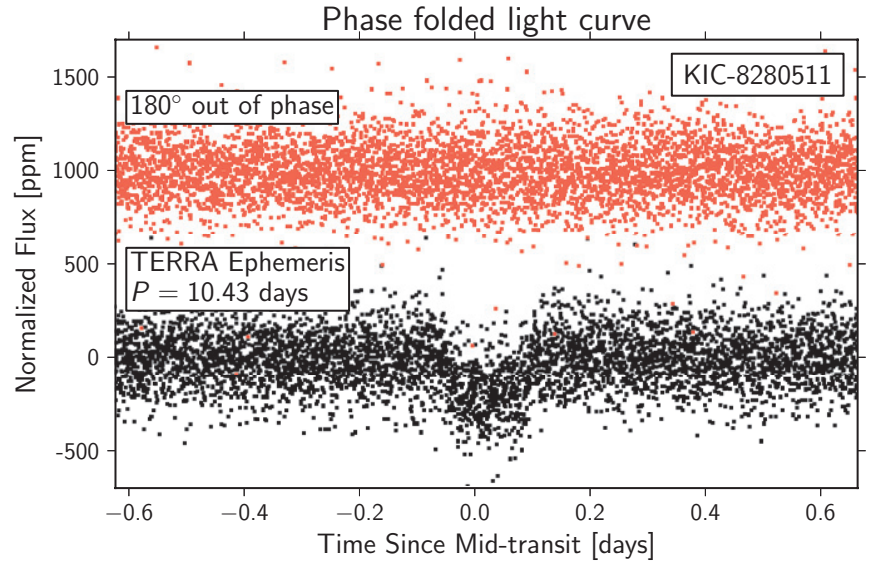

Figure 13. Phase-folded photometry of KIC-8280511 folded on the correct 10.43 day period found by TERRA. KOI- 1151.01 is listed with $P=5.22$ days in Batalha et al. (2012). If the transit was truly on the 5.22 day period, we should see a transit of equal depth 180 degrees out of phase. KOI-1151.01 is listed in Batalha et al. (2012) with half its true period.

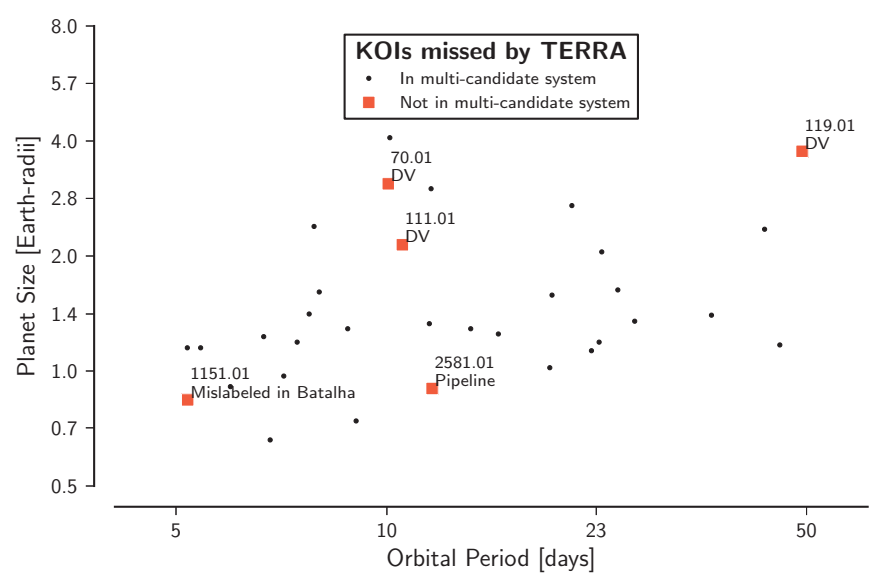

Figure 14. $P$ and $R_{P}$ for the 33 candidates present in Batalha et al. (2012) but not found by TERRA. The small symbols show the candidates in multi-planet systems. TERRA is blind to such candidates. The five larger symbols show the other failure modes of TERRA: 2581.01 failed due to a pipeline bug; 70.01, 111.01, and 119.01 did not pass DV; and TERRA missed KOI-1151.01 because it is listed in Batalha et al. (2012) with the incorrect period. Most of the missed planets have $R_{P}<1.4 R_{E}$.

pipeline to multis is nearly complete for $R_{P}>1.4 R_{E}$. TERRA has $>80 \%$ completeness for $R_{P}>1.4 R_{E}$ because planets in that size range with $P=5-50$ days around Best12k stars have high $\mathrm{S} / \mathrm{N}$. The Kepler pipeline should also be detecting these high $\mathrm{S} / \mathrm{N}$ candidates. Also, once a KOI is found, the Kepler team reprocesses the light curve for additional transits (Jason Rowe 2012, private communication). Due to this additional scrutiny, we believe that the Kepler completeness for multis is higher than for singles, all else being equal.

We then add $f_{\text {cell, Batalha }}$ to $f_{\text {cell }}$ computed in the previous section. We show occurrence computed using TERRA and Batalha et al. (2012) planets as a function of $P$ and $R_{P}$ in Figure 15 and as a function of only $R_{P}$ in Figure 16 . The 32 additional planets from Batalha et al. (2012) do not change the overall shape of the occurrence distribution: rising from 4.0 to $2.8 R_{E}$ and consistent with flat from 2.8 down to $1.0 R_{E}$.

H12 fit occurrence for $R_{P}>2 R_{E}$ with a power law,

$$
\frac{d f}{d \log R_{P}}=k_{R} R_{P}^{\alpha},
$$




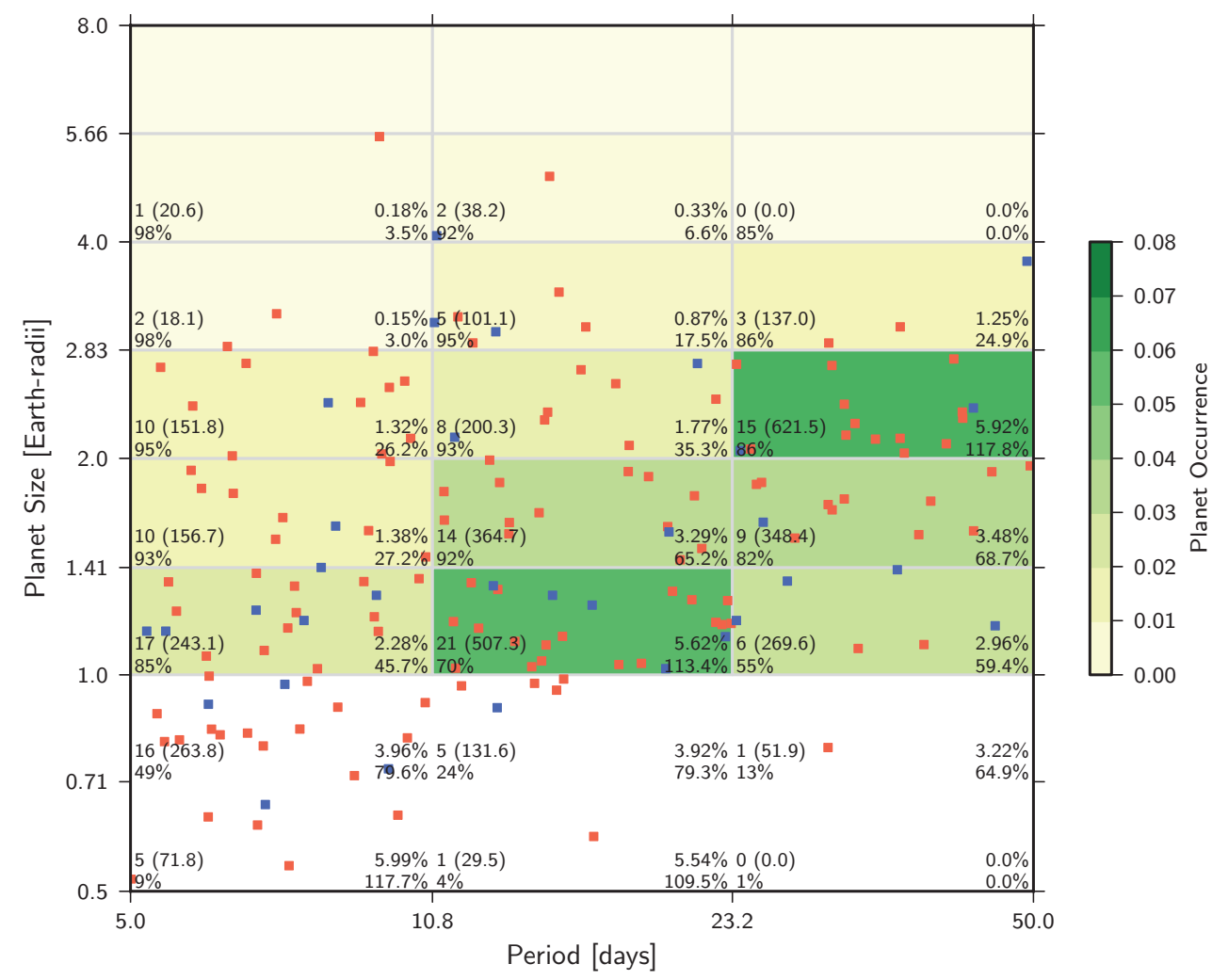

Figure 15. As in Figure 10, red points show 119 TERRA-detected planets. Blue points represent additional planets from Batalha et al. (2012). Most (28 out of 32 ) of these new candidates are planets in multi-candidate systems where TERRA successfully identifies the higher S/N candidate. We apply no completeness correction to these new planets, and we believe this is appropriate for $R_{P}>1.4 R_{E}$. We quote the following occurrence information for each cell: top left: number of planets (number of augmented planets), lower left: completeness, top right: fractional planet occurrence $f_{\text {cell }}$, bottom right: normalized planet occurrence $d^{2} f_{\text {cell }} / d \log P / d \log R_{P}$. We do not color cells where the completeness is less than $50 \%$ (i.e., the completeness correction is larger than a factor of two). The planet counts and occurrence values are for the combined TERRA and Batalha et al. (2012) sample. The completeness values are the same as in Figure 10.

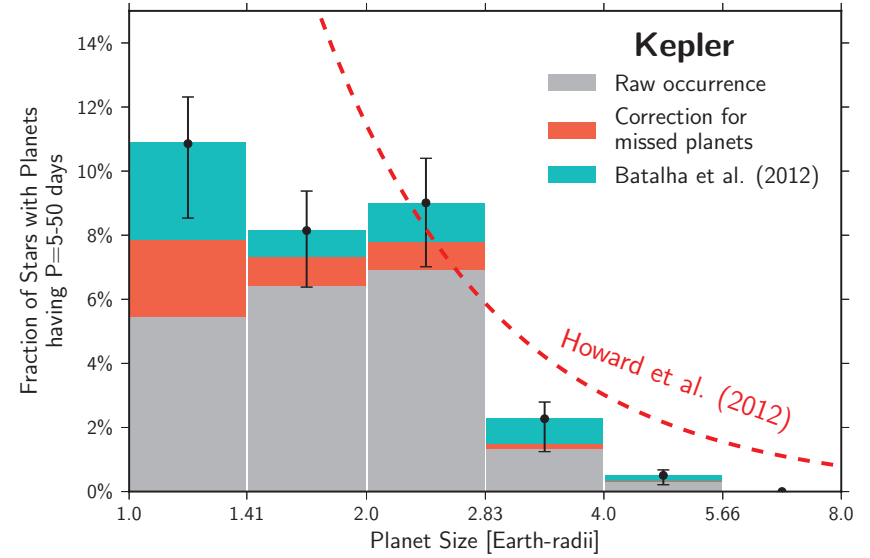

Figure 16. Same as Figure 11 with inclusion of planets in multi-planet systems. The blue regions represent the additional contribution to planet occurrence from the Batalha et al. (2012) planets. The addition of these new planets does not change the overall shape of the distribution. The dashed line is the power law fit to the planet size distribution in H12. The fit agrees qualitatively for $R_{P}>$ $2 R_{E}$, but not within errors. We expect the $\mathrm{H} 12$ fit to be $\sim 25 \%$ higher than our occurrence measurements since H12 included planets with $P<50$ days (not $P=5-50$ days). Additional discrepancies between occurrence in $\mathrm{H} 12$ and this work could stem from different characterizations of completeness, reliance on photometric vs. spectroscopic measurements of $R_{\star}$, and magnitude-limited, rather than noise-limited, samples.

finding $\alpha=-1.92 \pm 0.11$ and $k_{R}=2.9_{-0.4}^{+0.5}$ (Section 3.1 of H12). As a point of comparison, we plot the H12 power law over our combined occurrence distribution in Figure 16. The fit agrees qualitatively for $R_{P}>2 R_{E}$, but not within errors. We expect the $\mathrm{H} 12$ fit to be $\sim 25 \%$ higher than our occurrence measurements since $\mathrm{H} 12$ included planets with $P<50$ days (not $P=5-50$ days). Additional discrepancies could stem from different characterizations of completeness, reliance on photometric versus spectroscopic measurements of $R_{\star}$, and magnitude-limited, rather than noise-limited, samples.

\section{DISCUSSION}

\subsection{TERRA}

We implement in this work a new pipeline for the detection of transiting planets in Kepler photometry and apply it to a sample of 12,000 G and K-type dwarfs stars chosen to be among the most photometrically quiet of the Kepler target stars. These low-noise stars offer the best chance for the detection of small, Earth-size planets in the Kepler field and will one day be among the stars from which $\eta_{\oplus}$-the fraction of Sun-like stars bearing Earth-size planets in habitable zone orbits-is estimated. In this work, we focus on the close-in planets having orbital periods of 5-50 days and semi-major axes $\lesssim 0.25$ AU. Earth-size planets with these characteristics are statistically at the margins of detectability with the current $\sim 3 \mathrm{yr}$ of photometry in Kepler quarters Q1-Q12.

Our TERRA pipeline has two key features that enable confident measurement of the occurrence of close-in planets approaching Earth size. First, TERRA calibrates the Kepler photometry and searches for transit signals independent of the results from the Kepler mission's official pipeline. In some cases, TERRA calibration achieves superior noise suppression compared to the Pre-search Data Conditioning module of the official Kepler pipeline (Petigura \& Marcy 2012). The transit search algorithm 
in TERRA is efficient at detecting low $\mathrm{S} / \mathrm{N}$ transits in the calibrated light curves. This algorithm successfully rediscovers 82 of 86 stars bearing planets in Batalha et al. (2012). Recall that the current version of TERRA only detects the highest $\mathrm{S} / \mathrm{N}$ transit signal in each system. Thus, additional planets orbiting known hosts are not reported here. We report the occurrence of stars having one or more planets, not the mean number of planets per star as in H12 and elsewhere. Our pipeline also detects 37 planets not found in Batalha et al. (2012) (19 of which were not in the catalog of the Kepler team as of 2012 August 8), albeit with the benefit of 6 quarters of additional photometry for TERRA to search.

The second crucial feature of TERRA is that we have characterized its detection completeness via the injection and recovery of synthetic transits in real Kepler light curves from the Best $12 \mathrm{k}$ sample. This completeness study is crucial to our occurrence calculations because it allows us to statistically correct for incompleteness variations across the $P-R_{P}$ plane. While the Kepler Project has initiated a completeness study of the official pipeline (Christiansen et al. 2013), TERRA is the only pipeline for Kepler photometry whose detection completeness has been calibrated by injection and recovery tests. Prior to TERRA, occurrence calculations required one to assume that the Kepler planet detections were complete down to some $\mathrm{S} / \mathrm{N}$ limit, or to estimate completeness based on $\mathrm{S} / \mathrm{N}$ alone without empirical tests of the performance of the algorithms in the pipeline. For example, H12 made cuts in stellar brightness $(K p<15)$ and transit $\mathrm{S} / \mathrm{N}(>10$ in a single quarter of photometry) and restricted their search to planets larger than $2 R_{E}$ with orbital periods shorter than 50 days. These conservative cuts on the planet and star catalogs were driven by the unknown completeness of the official Kepler pipeline at low S/N. H12 applied two statistical corrections to convert their distribution of detected planets into an occurrence distribution. They corrected for non-transiting planets with a geometric $a / R_{\star}$ correction. They also computed the number of stars amenable to the detection (at $\mathrm{S} / \mathrm{N}>10$ in a single quarter) of each planet and considered only that number of stars in the occurrence calculation. H12 had no empirical way to determine the actual detection efficiency of the algorithms in the pipeline. Here, we apply the geometric $a / R_{\star}$ correction and correct for pipeline completeness across the $P-R_{P}$ plane by explicit tests of the TERRA pipeline efficiency, which naturally incorporates an $\mathrm{S} / \mathrm{N}$ threshold correction as in $\mathrm{H} 12$.

\subsection{Planet Occurrence}

$\mathrm{H} 12$ found that for close-in planets, the planet radius function rises steeply from Jupiter size to $2 R_{E}$. For smaller planets of $\sim 1-2 R_{E}$, occurrence was approximately constant in logarithmic $R_{P}$ bins, but $\mathrm{H} 12$ were skeptical of the result below $2 R_{E}$ because of unknown pipeline completeness and the small number statistics near $1 R_{E}$ in the Borucki et al. (2011b) planet catalog. In this work, we strongly confirm the power law rise in occurrence from 4 to $2 R_{E}$ using a superior assessment of completeness and nine times more photometry than in H12. Using TERRA, we can empirically and confidently compute occurrence down to $1 R_{E}$. Our key result is the plateau of planet occurrence for the size range $1-2.8 R_{E}$ for planets having orbital periods 5-50 days around Sun-like stars. In that size range of $1-2.8 R_{E}, 23 \%$ of stars have a planet orbiting with periods between 5 and 50 days. Including the multiple planets within each system, we find 0.28 planets per star within the size range 1-2.8 $R_{E}$ and with periods between 5 and 50 days. These results apply, of course, to the Kepler field, with its still unknown distribution of masses, ages, and metallicities in the Galactic disk.

As shown in Figure 10, TERRA detects many sub-Earth size planets $\left(<1.0 R_{E}\right)$. These sub-Earths appear in regions of low completeness, and, provocatively, may represent just the tip of the iceberg. A rich population of sub-Earths may await discovery given more photometry and continued pipeline improvements. With $8 \mathrm{yr}$ of total photometry in an extended Kepler mission (compared to $3 \mathrm{yr}$ here), the computational machinery of TERRA - including its light curve calibration, transit search, and completeness calibration-will enable a measurement of $\eta_{\oplus}$ for habitable zone orbits.

\subsection{Interpretation}

We are not the first to note the huge population of close-in planets smaller or less massive than Neptune. Using Doppler surveys, Howard et al. (2010) and Mayor et al. (2011) showed that the planet mass function rises steeply with decreasing mass, at least for close-in planets. In Kepler data, the excess of closein, small planets was obvious in the initial planet catalogs released by the Kepler Project (Borucki et al. 2011a, 2011b). H12 characterized the occurrence distribution of these small planets as a function of their size, orbital period, and host star temperature. These occurrence measurements, based on official Kepler planet catalogs, were refined and extended by Youdin (2011), Traub (2012), Dong \& Zhu (2012), Beaugé \& Nesvorný (2013), and others. Our contribution here shows a clear plateau in occurrence in the $1-2.8 R_{E}$ size range and certified by an independent search of Kepler photometry using a pipeline calibrated by injection and recovery tests. The onset of the plateau at $\sim 2.8 R_{E}$ suggests that there is a preferred size scale for the formation of close-in planets.

H12 and Youdin (2011) noted falling planet occurrence for periods shorter than $\sim 7$ days. We also observe declining planet occurrence for short orbital periods, but find that the transition occurs closer to $\sim 10$ days. We consider our period distribution to be in qualitative agreement with those of H12 and Youdin (2011). Planet formation and/or migration seems to discourage very close-in planets $(P \lesssim 10$ days $)$.

Close-in, small planets are now the most abundant planets detected by current transit and Doppler searches, yet they are absent from the solar system. The solar system is devoid of planets between 1 and $3.88 R_{E}$ (Earth and Neptune) and planets with periods less than Mercury's $(P<88.0$ days $)$. The formation mechanisms and possible subsequent migration of such planets are hotly debated. The population synthesis models of Ida \& Lin (2010) and Mordasini et al. (2009) suggest that they form near or beyond the ice line and then migrate quiescently in the protoplanetary disk. These models follow the growth and migration of planets over a wide range of parameters (from Jupiter mass down to Earth mass orbiting at distances out to $\sim 10$ AU) and they predict "deserts" of planet occurrence that are not detected.

More recently, Hansen \& Murray (2012) and Chiang \& Laughlin (2012) have argued for the in situ formation of close-in planets of Neptune size and smaller. In these models, close-in rocky planets of a few Earth masses form from protoplanetary disks more massive than the minimum mass solar nebula. Multiple planets per disk form commonly in these models and accretion is fast $\left(\sim 10^{5} \mathrm{yr}\right)$ and efficient due to the short dynamical timescales of close-in orbits. The rocky cores form before the protoplanetary disk has dissipated, accreting nebular gas that adds typically $\sim 3 \%$ to the mass of the planet (Chiang \& 
Laughlin 2012). But the small amounts of gas can significantly swell the radii of these otherwise rocky planets. For example, Adams et al. (2008) found that adding a $\mathrm{H} / \mathrm{He}$ gas envelope equivalent to $0.2 \%-20 \%$ of the mass of a solid $5 M_{E}$ planet increases the radius $8 \%-110 \%$ above the gas-free value.

We find the in situ model plausible because it naturally explains the large number of close, sub-Neptune-size planets, the high rate of planet multiplicity and nearly co-planar and circular orbits (Lissauer et al. 2011; Fang \& Margot 2012), and does not require tuning of planet migration models. Our result of a plateau in the planet size distribution for 1-2.8 $R_{E}$ with a sharp falloff in occurrence for larger planets along with decreasing occurrence for $P \lesssim 10$ days are two significant observed properties of planets around Sun-like stars that must be reproduced by models that form planets in situ or otherwise and by associated population synthesis models.

The in situ model seems supported by the sheer large occurrence of sub-Neptune-size planets within $0.25 \mathrm{AU}$. It seems unlikely that all such planets form beyond the snow line at $\sim 2 \mathrm{AU}$, which would require inward migration to within $0.25 \mathrm{AU}$, but not all the way into the star. Such models of formation beyond the snow line seem to require fine tuning of migration and parking mechanisms, as well as the tuning of available water or gas beyond $2 \mathrm{AU}$, while avoiding runaway gas accretion toward Jupiter masses. Still, in situ formation seems to require higher densities than those normally assumed in a minimum mass solar nebula (Chiang \& Laughlin 2012) in order to form the sub-Neptune planets before removal of the gas. If this in situ model is correct, we expect these sub-Neptune-size planets to be composed of rock plus $\mathrm{H}$ and $\mathrm{He}$, rather than rock plus water (Chiang \& Laughlin 2012). Thus, a test of the in situ mode of formation involves spectroscopic measurements of the chemical composition of the close-in sub-Neptunes.

The authors are indebted to Jon Jenkins, Peter Nugent, Howard Isaacson, Rea Kolbl, Eugene Chiang, Jason Rowe, and Stephen Bryson for productive and enlightening conversations that improved this work. We recognize the independent and complementary work of Fressin et al. (2013), who arrive at similar estimates of planet occurrence. We acknowledge salary support for Petigura by the National Science Foundation through the Graduate Research Fellowship Program. This research used resources of the National Energy Research Scientific Computing Center, which is supported by the Office of Science of the U.S. Department of Energy under contract No. DE-AC0205CH11231. This work made use of NASA's Astrophysics Data System Bibliographic Services as well as the NumPy (Oliphant 2007), SciPy (Jones et al. 2001), h5py (Collette 2008), IPython (Pérez \& Granger 2007), and Matplotlib (Hunter 2007) Python modules. Finally, we extend special thanks to those of Hawai' ian ancestry on whose sacred mountain of Mauna Kea we are privileged to be guests. Without their generous hospitality, the Keck observations presented herein would not have been possible.

\section{APPENDIX}

Table 2

Planet Candidates Identified with TERRA

\begin{tabular}{|c|c|c|c|c|c|c|c|c|c|c|c|c|c|c|c|c|}
\hline \multirow[b]{2}{*}{ KIC } & \multicolumn{8}{|c|}{ Light Curve Fit } & \multicolumn{3}{|c|}{ Stellar Parameters } & \multirow[b]{2}{*}{$\begin{array}{c}R_{P} \\
\left(R_{E}\right)\end{array}$} & \multirow[b]{2}{*}{$\sigma\left(R_{P}\right)$} & \multirow[b]{2}{*}{ Source $^{b}$} & \multirow[b]{2}{*}{ FP } & \multirow[b]{2}{*}{$\mathrm{B} 12$} \\
\hline & $\begin{array}{c}P \\
\text { (days) }\end{array}$ & $\begin{array}{c}t_{0}^{\mathrm{a}} \\
\text { (days) }\end{array}$ & $\begin{array}{l}\frac{R_{P}}{R_{\star}} \\
(\%)\end{array}$ & $\sigma\left(\frac{R_{P}}{R_{\star}}\right)$ & $\begin{array}{c}\tau \\
(\mathrm{hr})\end{array}$ & $\sigma(\tau)$ & $b$ & $\sigma(b)$ & $\begin{array}{l}T_{\text {eff }} \\
(\mathrm{K})\end{array}$ & $\begin{array}{l}\log g \\
(\mathrm{cgs}) \\
\end{array}$ & $\begin{array}{c}R_{\star} \\
\left(R_{\odot}\right)\end{array}$ & & & & & \\
\hline 2142522 & 13.323 & 67.043 & 0.98 & 0.34 & 2.32 & 0.51 & $<0.85$ & & 6046 & 4.40 & 1.04 & 1.11 & 0.39 & $\mathrm{P} 1$ & $\mathrm{Y}$ & $\mathrm{N}$ \\
\hline 2307415 & 13.122 & 66.396 & 1.26 & 0.13 & 1.90 & 0.12 & $<0.52$ & & 6133 & 4.38 & 1.14 & 1.57 & 0.16 & $\mathrm{~S}$ & $\mathrm{~N}$ & $\mathrm{Y}$ \\
\hline 2441495 & 12.493 & 71.457 & 2.38 & 0.24 & 1.13 & 0.03 & $<0.47$ & & 5192 & 4.56 & 0.76 & 1.99 & 0.20 & $\mathrm{~S}$ & $\mathrm{~N}$ & Y \\
\hline 2444412 & 14.911 & 74.337 & 3.38 & 0.34 & 4.24 & 0.31 & 0.93 & 0.01 & 5551 & 4.47 & 0.92 & 3.41 & 0.34 & $\mathrm{~S}$ & $\mathrm{~N}$ & $\mathrm{Y}$ \\
\hline 2571238 & 9.287 & 68.984 & 2.90 & 0.29 & 3.72 & 0.38 & 0.90 & 0.03 & 5544 & 4.50 & 0.89 & 2.82 & 0.28 & $\mathrm{~S}$ & $\mathrm{~N}$ & $\mathrm{Y}$ \\
\hline 2853446 & 7.373 & 70.613 & 1.39 & 0.14 & 0.74 & 0.06 & $<0.70$ & & 5969 & 4.37 & 1.10 & 1.65 & 0.17 & $\mathrm{~S}$ & $\mathrm{~N}$ & $\mathrm{Y}$ \\
\hline 3098810 & 40.811 & 75.673 & 1.98 & 0.20 & 1.45 & 0.12 & $<0.70$ & & 6071 & 4.31 & 1.27 & 2.75 & 0.28 & $\mathrm{~S}$ & $\mathrm{~N}$ & $\mathrm{Y}$ \\
\hline 3120904 & 42.915 & 72.866 & 1.15 & 0.12 & 2.96 & 0.36 & $<0.66$ & & 6151 & 4.31 & 1.26 & 1.59 & 0.16 & $\mathrm{~S}$ & & $\mathrm{~N}$ \\
\hline 3342794 & 14.172 & 75.591 & 1.40 & 0.14 & 1.31 & 0.13 & $<0.55$ & & 5900 & 4.35 & 1.10 & 1.68 & 0.17 & $\mathrm{~S}$ & $\mathrm{~N}$ & $\mathrm{Y}$ \\
\hline 3442055 & 29.619 & 66.681 & 1.57 & 0.16 & 2.48 & 0.15 & $<0.57$ & & 5624 & 4.41 & 1.01 & 1.72 & 0.17 & $\mathrm{~S}$ & $\mathrm{~N}$ & $\mathrm{Y}$ \\
\hline 3531558 & 24.994 & 71.674 & 1.49 & 0.15 & 2.93 & 0.12 & $<0.53$ & & 5808 & 4.35 & 1.14 & 1.85 & 0.19 & S & $\mathrm{N}$ & $\mathrm{Y}$ \\
\hline 3545135 & 8.483 & 65.973 & 0.81 & 0.08 & 1.44 & 0.10 & $<0.57$ & & 5794 & 4.40 & 1.02 & 0.90 & 0.09 & $\mathrm{~S}$ & $\mathrm{~N}$ & $\mathrm{~N}$ \\
\hline 3835670 & 14.558 & 78.084 & 2.86 & 0.29 & 3.89 & 0.22 & 0.31 & 0.19 & 5722 & 4.14 & 1.58 & 4.93 & 0.49 & $\mathrm{~S}$ & $\mathrm{~N}$ & $\mathrm{Y}$ \\
\hline 3839488 & 11.131 & 67.370 & 1.36 & 0.14 & 1.97 & 0.11 & $<0.50$ & & 5991 & 4.36 & 1.11 & 1.64 & 0.17 & $\mathrm{~S}$ & $\mathrm{~N}$ & $\mathrm{Y}$ \\
\hline 3852655 & 11.629 & 65.817 & 0.85 & 0.30 & 1.49 & 0.23 & $<0.79$ & & 5822 & 4.36 & 1.05 & 0.97 & 0.34 & $\mathrm{P} 1$ & $\mathrm{~N}$ & $\mathrm{~N}$ \\
\hline 3942670 & 33.416 & 70.911 & 1.48 & 0.15 & 3.99 & 0.24 & $<0.63$ & & 6012 & 4.28 & 1.32 & 2.13 & 0.21 & $\mathrm{~S}$ & $\mathrm{~N}$ & $\mathrm{Y}$ \\
\hline 4043190 & 6.401 & 69.883 & 1.07 & 0.11 & 1.35 & 0.08 & $<0.65$ & & 5302 & 3.83 & 2.45 & 2.86 & 0.29 & $\mathrm{~S}$ & $\mathrm{~N}$ & $\mathrm{Y}$ \\
\hline 4049901 & 16.291 & 65.115 & 0.64 & 0.23 & 1.72 & 0.16 & $<0.68$ & & 5250 & 4.48 & 0.85 & 0.60 & 0.21 & $\mathrm{P} 1$ & $\mathrm{~N}$ & $\mathrm{Y}$ \\
\hline 4548011 & 6.284 & 66.198 & 0.60 & 0.06 & 2.23 & 0.64 & $<0.49$ & & 5991 & 4.30 & 1.26 & 0.83 & 0.09 & $\mathrm{~S}$ & & $\mathrm{~N}$ \\
\hline 4644604 & 14.486 & 64.550 & 2.03 & 0.21 & 1.49 & 0.15 & $<0.59$ & & 5739 & 4.34 & 1.04 & 2.32 & 0.23 & $\mathrm{~S}$ & $\mathrm{~N}$ & $\mathrm{Y}$ \\
\hline 4770174 & 6.096 & 67.600 & 0.57 & 0.20 & 3.08 & 0.44 & $<0.75$ & & 6013 & 4.44 & 1.01 & 0.63 & 0.22 & $\mathrm{P} 1$ & $\mathrm{~N}$ & $\mathrm{~N}$ \\
\hline 4827723 & 7.239 & 68.024 & 1.63 & 0.17 & 2.20 & 0.37 & 0.70 & 0.12 & 5392 & 4.52 & 0.87 & 1.54 & 0.16 & $\mathrm{~S}$ & $\mathrm{~N}$ & $\mathrm{Y}$ \\
\hline 4914423 & 15.965 & 75.182 & 2.17 & 0.22 & 3.14 & 0.28 & 0.71 & 0.06 & 5904 & 4.27 & 1.29 & 3.05 & 0.31 & S & $\mathrm{N}$ & $\mathrm{Y}$ \\
\hline 4914566 & 22.241 & 77.736 & 0.83 & 0.29 & 3.76 & 0.36 & $<0.73$ & & 5974 & 4.22 & 1.31 & 1.18 & 0.41 & $\mathrm{P} 1$ & $\mathrm{Y}$ & $\mathrm{N}$ \\
\hline 5009743 & 41.699 & 102.563 & 1.94 & 0.20 & 2.87 & 0.30 & $<0.70$ & & 5937 & 4.35 & 1.09 & 2.32 & 0.23 & $\mathrm{~S}$ & $\mathrm{~N}$ & $\mathrm{Y}$ \\
\hline 5042210 & 12.147 & 64.535 & 0.81 & 0.08 & 3.22 & 0.21 & $<0.56$ & & 6007 & 4.27 & 1.31 & 1.16 & 0.12 & $\mathrm{~S}$ & $\mathrm{~N}$ & Y \\
\hline 5094751 & 6.482 & 68.943 & 1.69 & 0.17 & 2.51 & 0.31 & 0.69 & 0.13 & 5929 & 4.37 & 1.10 & 2.02 & 0.20 & $\mathrm{~S}$ & $\mathrm{~N}$ & $\mathrm{Y}$ \\
\hline 5096590 & 29.610 & 70.332 & 1.00 & 0.35 & 2.60 & 0.20 & $<0.67$ & & 5623 & 4.63 & 0.73 & 0.79 & 0.28 & $\mathrm{P} 1$ & & $\mathrm{~N}$ \\
\hline 5121511 & 30.996 & 93.790 & 2.35 & 0.24 & 1.40 & 0.06 & $<0.47$ & & 5217 & 4.53 & 0.84 & 2.15 & 0.22 & $\mathrm{~S}$ & $\mathrm{~N}$ & $\mathrm{Y}$ \\
\hline 5308537 & 14.265 & 76.217 & 0.76 & 0.08 & 1.92 & 0.53 & $<0.87$ & & 5831 & 4.29 & 1.26 & 1.05 & 0.11 & $\mathrm{~S}$ & & $\mathrm{~N}$ \\
\hline
\end{tabular}


Table 2

(Continued)

\begin{tabular}{|c|c|c|c|c|c|c|c|c|c|c|c|c|c|c|c|c|}
\hline \multirow[b]{2}{*}{ KIC } & \multicolumn{8}{|c|}{ Light Curve Fit } & \multicolumn{3}{|c|}{ Stellar Parameters } & \multirow[b]{2}{*}{$\begin{array}{c}R_{P} \\
\left(R_{E}\right)\end{array}$} & \multirow[b]{2}{*}{$\sigma\left(R_{P}\right)$} & \multirow[b]{2}{*}{ Source $^{b}$} & \multirow[b]{2}{*}{$\mathrm{FP}$} & \multirow[b]{2}{*}{ B12 } \\
\hline & $\begin{array}{c}P \\
\text { (days) }\end{array}$ & $\begin{array}{c}t_{0}^{\mathrm{a}} \\
\text { (days) }\end{array}$ & $\begin{array}{l}\frac{R_{P}}{R_{\star}} \\
(\%)\end{array}$ & $\sigma\left(\frac{R_{P}}{R_{\star}}\right)$ & $\begin{array}{c}\tau \\
(\mathrm{hr})\end{array}$ & $\sigma(\tau)$ & $b$ & $\sigma(b)$ & $\begin{array}{l}T_{\text {eff }} \\
(\mathrm{K})\end{array}$ & $\begin{array}{l}\log g \\
(\mathrm{cgs})\end{array}$ & $\begin{array}{c}R_{\star} \\
\left(R_{\odot}\right)\end{array}$ & & & & & \\
\hline 5561278 & 20.310 & 79.826 & 1.16 & 0.12 & 2.73 & 0.15 & $<0.47$ & & 6161 & 4.38 & 1.14 & 1.45 & 0.15 & $S$ & $\mathrm{~N}$ & $\mathrm{Y}$ \\
\hline 5613330 & 23.449 & 69.031 & 1.68 & 0.17 & 4.44 & 0.28 & 0.25 & 0.22 & 6080 & 4.20 & 1.48 & 2.70 & 0.27 & S & $\mathrm{N}$ & $\mathrm{Y}$ \\
\hline 5652893 & 14.010 & 65.959 & 1.15 & 0.13 & 1.98 & 0.49 & $<0.85$ & & 5150 & 4.55 & 0.78 & 0.97 & 0.11 & S & $\mathrm{N}$ & $\mathrm{N}$ \\
\hline 5702939 & 18.398 & 77.934 & 1.09 & 0.38 & 2.45 & 0.27 & $<0.54$ & & 5634 & 4.47 & 0.87 & 1.04 & 0.36 & $\mathrm{P} 1$ & $\mathrm{~N}$ & $\mathrm{~N}$ \\
\hline 5735762 & 9.674 & 68.009 & 2.73 & 0.27 & 1.61 & 0.06 & $<0.47$ & & 5195 & 4.53 & 0.84 & 2.51 & 0.25 & $\mathrm{~S}$ & $\mathrm{~N}$ & $\mathrm{Y}$ \\
\hline 5866724 & 5.860 & 65.040 & 1.65 & 0.17 & 2.01 & 0.05 & 0.22 & 0.14 & 6109 & 4.29 & 1.31 & 2.37 & 0.24 & S & $\mathrm{N}$ & $\mathrm{Y}$ \\
\hline 5959719 & 6.738 & 66.327 & 0.99 & 0.10 & 1.30 & 0.21 & $<0.76$ & & 5166 & 4.56 & 0.77 & 0.83 & 0.09 & $\mathrm{~S}$ & $\mathrm{~N}$ & $\mathrm{Y}$ \\
\hline 6071903 & 24.308 & 87.054 & 2.28 & 0.23 & 1.29 & 0.05 & $<0.27$ & & 5296 & 4.55 & 0.83 & 2.06 & 0.21 & S & $\mathrm{N}$ & $\mathrm{Y}$ \\
\hline 6197215 & 10.613 & 68.691 & 1.23 & 0.13 & 0.48 & 0.06 & $<0.80$ & & 5933 & 4.39 & 1.09 & 1.46 & 0.15 & S & $\mathrm{N}$ & $\mathrm{N}$ \\
\hline 6289257 & 19.675 & 69.903 & 1.33 & 0.13 & 1.96 & 0.21 & 0.35 & 0.27 & 6023 & 4.36 & 1.11 & 1.61 & 0.16 & S & $\mathrm{N}$ & $\mathrm{Y}$ \\
\hline 6291837 & 35.596 & 84.942 & 2.45 & 0.25 & 3.18 & 0.42 & $<0.71$ & & 6165 & 4.38 & 1.14 & 3.05 & 0.31 & $S$ & $\mathrm{~N}$ & $\mathrm{Y}$ \\
\hline 6356692 & 11.392 & 74.555 & 0.66 & 0.23 & 2.74 & 0.31 & $<0.73$ & & 5420 & 4.03 & 1.64 & 1.19 & 0.42 & $\mathrm{P} 1$ & $\mathrm{~N}$ & $\mathrm{~N}$ \\
\hline 6365156 & 10.214 & 73.050 & 1.57 & 0.16 & 2.83 & 0.09 & $<0.42$ & & 5852 & 4.28 & 1.25 & 2.13 & 0.21 & $\mathrm{~S}$ & $\mathrm{~N}$ & $\mathrm{Y}$ \\
\hline 6442340 & 13.137 & 76.973 & 1.38 & 0.14 & 2.41 & 0.13 & $<0.55$ & & 5764 & 4.38 & 1.08 & 1.63 & 0.16 & S & $\mathrm{N}$ & $\mathrm{Y}$ \\
\hline 6521045 & 12.816 & 68.772 & 1.37 & 0.14 & 3.42 & 0.34 & 0.40 & 0.19 & 5874 & 4.29 & 1.24 & 1.85 & 0.19 & S & $\mathrm{N}$ & $\mathrm{Y}$ \\
\hline 6523351 & 6.067 & 69.128 & 0.72 & 0.25 & 1.02 & 0.20 & $<0.89$ & & 5489 & 4.15 & 1.35 & 1.06 & 0.37 & $\mathrm{P} 1$ & & $\mathrm{~N}$ \\
\hline 6605493 & 9.310 & 69.379 & 0.99 & 0.10 & 1.80 & 0.16 & $<0.62$ & & 5805 & 4.36 & 1.12 & 1.20 & 0.12 & $\mathrm{~S}$ & $\mathrm{~N}$ & $\mathrm{Y}$ \\
\hline 6607357 & 7.700 & 67.390 & 0.85 & 0.30 & 2.72 & 0.45 & 0.55 & 0.22 & 5592 & 4.51 & 0.91 & 0.84 & 0.29 & $\mathrm{P} 1$ & $\mathrm{~N}$ & $\mathrm{~N}$ \\
\hline 6707835 & 22.248 & 84.881 & 2.25 & 0.23 & 1.97 & 0.07 & $<0.43$ & & 5619 & 4.44 & 0.99 & 2.42 & 0.24 & $\mathrm{~S}$ & $\mathrm{~N}$ & $\mathrm{Y}$ \\
\hline 6716545 & 13.910 & 75.855 & 0.84 & 0.30 & 3.29 & 0.68 & $<0.85$ & & 6044 & 4.30 & 1.12 & 1.03 & 0.36 & $\mathrm{P} 1$ & $\mathrm{~N}$ & $\mathrm{~N}$ \\
\hline 6803202 & 21.061 & 76.591 & 1.58 & 0.16 & 2.64 & 0.08 & $<0.36$ & & 5719 & 4.40 & 1.03 & 1.77 & 0.18 & $\mathrm{~S}$ & $\mathrm{~N}$ & $\mathrm{Y}$ \\
\hline 6851425 & 11.120 & 72.744 & 2.29 & 0.23 & 1.71 & 0.12 & $<0.62$ & & 5071 & 4.59 & 0.72 & 1.80 & 0.18 & S & $\mathrm{N}$ & $\mathrm{Y}$ \\
\hline 6922710 & 23.127 & 78.663 & 1.01 & 0.11 & 2.57 & 0.47 & $<0.78$ & & 5929 & 4.40 & 1.07 & 1.18 & 0.12 & $\mathrm{~S}$ & $\mathrm{~N}$ & $\mathrm{Y}$ \\
\hline 7021534 & 9.066 & 68.147 & 1.42 & 0.50 & 0.81 & 0.05 & $<0.55$ & & 5848 & 4.55 & 0.87 & 1.35 & 0.47 & $\mathrm{P} 1$ & Y & $\mathrm{N}$ \\
\hline 7033671 & 9.490 & 66.961 & 1.48 & 0.15 & 1.81 & 0.09 & $<0.46$ & & 5679 & 4.29 & 1.26 & 2.03 & 0.20 & $\mathrm{~S}$ & $\mathrm{~N}$ & $\mathrm{Y}$ \\
\hline 7211221 & 5.621 & 69.903 & 1.21 & 0.12 & 1.23 & 0.11 & $<0.53$ & & 5634 & 4.44 & 0.93 & 1.23 & 0.12 & $S$ & $\mathrm{~N}$ & $\mathrm{Y}$ \\
\hline 7219825 & 17.233 & 68.084 & 2.06 & 0.21 & 2.27 & 0.13 & $<0.22$ & & 6089 & 4.36 & 1.13 & 2.54 & 0.25 & $\mathrm{~S}$ & $\mathrm{~N}$ & $\mathrm{Y}$ \\
\hline 7345248 & 5.665 & 69.338 & 0.63 & 0.22 & 2.81 & 0.43 & $<0.61$ & & 5656 & 4.27 & 1.19 & 0.81 & 0.29 & P2 & & $\mathrm{N}$ \\
\hline 7419318 & 18.736 & 73.153 & 2.13 & 0.22 & 1.47 & 0.11 & $<0.61$ & & 5187 & 4.54 & 0.81 & 1.89 & 0.19 & $\mathrm{~S}$ & $\mathrm{~N}$ & $\mathrm{Y}$ \\
\hline 7466863 & 11.971 & 68.173 & 1.83 & 0.64 & 0.98 & 0.03 & $<0.39$ & & 6035 & 4.12 & 1.45 & 2.89 & 1.01 & $\mathrm{P} 1$ & $\mathrm{Y}$ & $\mathrm{N}$ \\
\hline 7582689 & 11.921 & 70.475 & 0.75 & 0.26 & 5.84 & 1.24 & $<0.95$ & & 6022 & 4.04 & 1.64 & 1.34 & 0.47 & $\mathrm{P} 1$ & & $\mathrm{~N}$ \\
\hline 7668663 & 6.498 & 69.012 & 1.44 & 0.15 & 1.85 & 0.34 & 0.79 & 0.09 & 5725 & 4.33 & 1.14 & 1.79 & 0.18 & $\mathrm{~S}$ & $\mathrm{~N}$ & $\mathrm{Y}$ \\
\hline 7700622 & 35.585 & 86.426 & 2.83 & 0.28 & 1.99 & 0.10 & $<0.46$ & & 4787 & 4.62 & 0.69 & 2.13 & 0.21 & $\mathrm{~S}$ & $\mathrm{~N}$ & $\mathrm{Y}$ \\
\hline 7762723 & 9.887 & 72.539 & 0.73 & 0.26 & 2.21 & 0.39 & $<0.65$ & & 5501 & 4.58 & 0.80 & 0.64 & 0.22 & $\mathrm{P} 1$ & $\mathrm{Y}$ & $\mathrm{N}$ \\
\hline 7810483 & 29.921 & 79.024 & 1.71 & 0.60 & 1.58 & 0.11 & $<0.58$ & & 5893 & 4.52 & 0.91 & 1.70 & 0.59 & $\mathrm{P} 1$ & $\mathrm{Y}$ & $\mathrm{N}$ \\
\hline 7906739 & 7.015 & 69.903 & 0.90 & 0.31 & 2.43 & 0.32 & $<0.68$ & & 5652 & 4.53 & 0.81 & 0.80 & 0.28 & P1 & $\mathrm{Y}$ & $\mathrm{N}$ \\
\hline 7906892 & 8.849 & 72.797 & 0.58 & 0.07 & 8.03 & 3.69 & 0.89 & 0.12 & 6095 & 4.35 & 1.14 & 0.72 & 0.08 & $\mathrm{~S}$ & & $\mathrm{~N}$ \\
\hline 7918652 & 11.456 & 69.316 & 0.79 & 0.28 & 2.45 & 0.47 & $<0.81$ & & 5809 & 4.25 & 1.19 & 1.02 & 0.36 & $\mathrm{P} 1$ & $\mathrm{~N}$ & $\mathrm{~N}$ \\
\hline 8008067 & 15.771 & 70.584 & 2.22 & 0.22 & 3.32 & 0.31 & 0.67 & 0.08 & 5594 & 4.37 & 1.10 & 2.66 & 0.27 & $\mathrm{~S}$ & $\mathrm{~N}$ & $\mathrm{Y}$ \\
\hline 8009496 & 38.476 & 83.567 & 1.88 & 0.66 & 1.72 & 0.14 & $<0.70$ & & 5833 & 4.54 & 0.85 & 1.74 & 0.61 & $\mathrm{P} 1$ & $\mathrm{Y}$ & $\mathrm{N}$ \\
\hline 8073705 & 10.601 & 65.967 & 0.75 & 0.08 & 2.29 & 0.36 & $<0.80$ & & 6086 & 4.36 & 1.12 & 0.91 & 0.10 & $\mathrm{~S}$ & & $\mathrm{~N}$ \\
\hline 8077137 & 15.090 & 78.772 & 0.78 & 0.08 & 2.47 & 0.65 & $<0.85$ & & 6179 & 4.37 & 1.16 & 0.99 & 0.10 & $\mathrm{~S}$ & $\mathrm{~N}$ & $\mathrm{Y}$ \\
\hline 8081187 & 37.323 & 85.828 & 1.67 & 0.59 & 3.58 & 0.39 & $<0.62$ & & 6030 & 4.55 & 0.86 & 1.57 & 0.55 & $\mathrm{P} 1$ & $\mathrm{Y}$ & $\mathrm{N}$ \\
\hline 8087812 & 27.211 & 65.360 & 1.01 & 0.10 & 5.01 & 0.57 & $<0.67$ & & 5985 & 4.17 & 1.41 & 1.55 & 0.16 & $\mathrm{~S}$ & & $\mathrm{~N}$ \\
\hline 8242434 & 44.964 & 77.565 & 2.58 & 0.26 & 2.58 & 0.15 & 0.31 & 0.22 & 4692 & 4.63 & 0.68 & 1.92 & 0.19 & $\mathrm{~S}$ & $\mathrm{~N}$ & $\mathrm{Y}$ \\
\hline 8280511 & 10.435 & 67.826 & 1.37 & 0.14 & 1.70 & 0.12 & $<0.58$ & & 5522 & 4.45 & 0.91 & 1.36 & 0.14 & $\mathrm{~S}$ & & $\mathrm{~N}$ \\
\hline 8323753 & 6.714 & 67.308 & 1.97 & 0.69 & 1.33 & 0.05 & $<0.52$ & & 5817 & 4.23 & 1.26 & 2.71 & 0.95 & $\mathrm{P} 1$ & $\mathrm{Y}$ & $\mathrm{N}$ \\
\hline 8349582 & 11.523 & 64.959 & 2.14 & 0.22 & 2.41 & 0.21 & 0.61 & 0.09 & 5668 & 4.23 & 1.35 & 3.15 & 0.32 & $\mathrm{~S}$ & $\mathrm{~N}$ & $\mathrm{Y}$ \\
\hline 8429668 & 5.007 & 67.696 & 0.73 & 0.26 & 1.66 & 0.47 & 0.62 & 0.29 & 5034 & 4.63 & 0.65 & 0.52 & 0.18 & $\mathrm{P} 2$ & & $\mathrm{~N}$ \\
\hline 8480285 & 29.667 & 92.687 & 2.43 & 0.25 & 4.72 & 0.42 & 0.48 & 0.14 & 5960 & 4.36 & 1.09 & 2.89 & 0.29 & $\mathrm{~S}$ & $\mathrm{~N}$ & $\mathrm{Y}$ \\
\hline 8494617 & 22.923 & 66.640 & 1.06 & 0.11 & 3.72 & 0.36 & $<0.50$ & & 5905 & 4.36 & 1.10 & 1.27 & 0.13 & $S$ & $\mathrm{~N}$ & $\mathrm{Y}$ \\
\hline 8560804 & 31.976 & 66.803 & 0.99 & 0.35 & 4.96 & 0.48 & $<0.65$ & & 5878 & 4.44 & 1.01 & 1.09 & 0.38 & $\mathrm{P} 1$ & $\mathrm{~N}$ & $\mathrm{~N}$ \\
\hline 8611832 & 22.597 & 73.431 & 1.08 & 0.11 & 3.15 & 0.17 & $<0.58$ & & 5577 & 4.37 & 0.99 & 1.17 & 0.12 & $\mathrm{~S}$ & $\mathrm{~N}$ & $\mathrm{Y}$ \\
\hline 8628758 & 14.374 & 71.204 & 1.99 & 0.20 & 4.76 & 0.68 & $<0.91$ & & 5773 & 4.40 & 1.04 & 2.26 & 0.23 & S & $\mathrm{N}$ & $\mathrm{Y}$ \\
\hline 8631504 & 14.820 & 66.409 & 1.20 & 0.12 & 2.02 & 0.25 & $<0.63$ & & 4828 & 4.60 & 0.72 & 0.95 & 0.10 & S & $\mathrm{N}$ & $\mathrm{Y}$ \\
\hline 8644365 & 19.917 & 72.354 & 1.07 & 0.11 & 3.37 & 0.55 & $<0.85$ & & 6054 & 4.39 & 1.12 & 1.31 & 0.13 & S & & $\mathrm{N}$ \\
\hline 8804455 & 7.597 & 64.958 & 1.14 & 0.12 & 2.55 & 0.47 & 0.74 & 0.11 & 5715 & 4.38 & 1.07 & 1.33 & 0.14 & S & $\mathrm{N}$ & $\mathrm{Y}$ \\
\hline 8805348 & 29.907 & 78.383 & 2.36 & 0.24 & 3.20 & 0.39 & 0.66 & 0.12 & 5739 & 4.34 & 1.05 & 2.69 & 0.27 & $\mathrm{~S}$ & $\mathrm{~N}$ & $\mathrm{Y}$ \\
\hline 8822366 & 30.864 & 65.012 & 1.41 & 0.14 & 4.16 & 0.20 & $<0.62$ & & 6089 & 4.35 & 1.14 & 1.76 & 0.18 & $\mathrm{~S}$ & $\mathrm{~N}$ & $\mathrm{Y}$ \\
\hline 8827575 & 10.129 & 68.997 & 0.84 & 0.30 & 1.94 & 0.18 & $<0.63$ & & 5284 & 4.45 & 0.89 & 0.82 & 0.29 & $\mathrm{P} 1$ & & $\mathrm{~N}$ \\
\hline 8866102 & 17.834 & 114.225 & 1.66 & 0.17 & 2.28 & 0.06 & $<0.39$ & & 6178 & 4.37 & 1.15 & 2.08 & 0.21 & S & $\mathrm{N}$ & $\mathrm{Y}$ \\
\hline 8962094 & 30.865 & 75.052 & 2.09 & 0.21 & 1.37 & 0.10 & $<0.61$ & & 5739 & 4.34 & 1.04 & 2.38 & 0.24 & S & $\mathrm{N}$ & $\mathrm{Y}$ \\
\hline 8972058 & 8.991 & 69.746 & 2.01 & 0.20 & 2.10 & 0.11 & $<0.52$ & & 5979 & 4.38 & 1.09 & 2.39 & 0.24 & S & $\mathrm{N}$ & $\mathrm{Y}$ \\
\hline
\end{tabular}


Table 2

(Continued)

\begin{tabular}{|c|c|c|c|c|c|c|c|c|c|c|c|c|c|c|c|c|}
\hline \multirow[b]{2}{*}{$\mathrm{KIC}$} & \multicolumn{8}{|c|}{ Light Curve Fit } & \multicolumn{3}{|c|}{ Stellar Parameters } & \multirow[b]{2}{*}{$\begin{array}{c}R_{P} \\
\left(R_{E}\right)\end{array}$} & \multirow[b]{2}{*}{$\sigma\left(R_{P}\right)$} & \multirow[b]{2}{*}{ Source $^{b}$} & \multirow[b]{2}{*}{ FP } & \multirow[b]{2}{*}{ B12 } \\
\hline & $\begin{array}{c}P \\
\text { (days) }\end{array}$ & $\begin{array}{c}t_{0}^{\mathrm{a}} \\
\text { (days) }\end{array}$ & $\begin{array}{l}\frac{R_{P}}{R_{\star}} \\
(\%)\end{array}$ & $\sigma\left(\frac{R_{P}}{R_{\star}}\right)$ & $\begin{array}{c}\tau \\
(\mathrm{hr})\end{array}$ & $\sigma(\tau)$ & $b$ & $\sigma(b)$ & $\begin{array}{l}T_{\text {eff }} \\
(\mathrm{K})\end{array}$ & $\begin{array}{l}\log g \\
(\operatorname{cgs})\end{array}$ & $\begin{array}{c}R_{\star} \\
\left(R_{\odot}\right)\end{array}$ & & & & & \\
\hline 9006186 & 5.453 & 67.152 & 0.85 & 0.09 & 1.07 & 0.08 & $<0.61$ & & 5404 & 4.53 & 0.87 & 0.81 & 0.08 & $\mathrm{~S}$ & $\mathrm{~N}$ & $\mathrm{Y}$ \\
\hline 9086251 & 6.892 & 64.632 & 0.87 & 0.09 & 0.93 & 0.10 & $<0.68$ & & 6044 & 4.22 & 1.45 & 1.38 & 0.14 & S & $\mathrm{N}$ & $\mathrm{Y}$ \\
\hline 9139084 & 5.836 & 67.853 & 2.07 & 0.21 & 1.06 & 0.03 & $<0.41$ & & 5411 & 4.53 & 0.85 & 1.92 & 0.19 & S & $\mathrm{N}$ & $\mathrm{Y}$ \\
\hline 9226339 & 21.461 & 65.230 & 1.10 & 0.11 & 1.66 & 0.16 & $<0.70$ & & 5807 & 4.28 & 1.25 & 1.50 & 0.15 & S & & $\mathrm{N}$ \\
\hline 9288237 & 7.491 & 68.329 & 0.52 & 0.18 & 3.01 & 0.73 & $<0.84$ & & 5946 & 4.44 & 0.96 & 0.54 & 0.19 & $\mathrm{P} 1$ & & $\mathrm{~N}$ \\
\hline 9491832 & 49.565 & 103.693 & 1.14 & 0.12 & 6.43 & 1.49 & 0.74 & 0.25 & 5821 & 4.15 & 1.57 & 1.95 & 0.21 & $\mathrm{~S}$ & & $\mathrm{~N}$ \\
\hline 9549648 & 5.992 & 69.883 & 1.46 & 0.15 & 1.80 & 0.45 & 0.89 & 0.06 & 6165 & 4.38 & 1.14 & 1.82 & 0.19 & $S$ & $\mathrm{~N}$ & $\mathrm{Y}$ \\
\hline 9704384 & 5.509 & 65.285 & 1.35 & 0.14 & 1.85 & 0.26 & $<0.75$ & & 5448 & 4.50 & 0.91 & 1.35 & 0.14 & $S$ & $\mathrm{~N}$ & $\mathrm{Y}$ \\
\hline 9716028 & 17.373 & 71.258 & 0.82 & 0.08 & 2.46 & 0.27 & $<0.73$ & & 6119 & 4.37 & 1.15 & 1.03 & 0.11 & S & & $\mathrm{N}$ \\
\hline 9717943 & 6.110 & 69.903 & 0.72 & 0.08 & 1.80 & 0.87 & 0.79 & 0.20 & 5968 & 4.30 & 1.27 & 1.00 & 0.11 & S & $\mathrm{N}$ & $\mathrm{Y}$ \\
\hline 9886361 & 7.031 & 67.464 & 0.88 & 0.09 & 3.04 & 0.21 & $<0.42$ & & 6090 & 4.39 & 1.13 & 1.08 & 0.11 & S & $\mathrm{N}$ & $\mathrm{N}$ \\
\hline 10055126 & 9.176 & 71.722 & 1.33 & 0.13 & 2.43 & 0.24 & $<0.48$ & & 5905 & 4.36 & 1.10 & 1.59 & 0.16 & S & $\mathrm{N}$ & $\mathrm{Y}$ \\
\hline 10130039 & 12.758 & 66.961 & 1.19 & 0.12 & 2.19 & 0.07 & $<0.38$ & & 5828 & 4.42 & 1.01 & 1.31 & 0.13 & S & $\mathrm{N}$ & $\mathrm{Y}$ \\
\hline 10136549 & 9.693 & 65.809 & 1.14 & 0.12 & 3.44 & 0.44 & 0.57 & 0.20 & 5684 & 4.13 & 1.59 & 1.98 & 0.20 & S & $\mathrm{N}$ & $\mathrm{Y}$ \\
\hline 10212441 & 15.044 & 66.211 & 0.95 & 0.10 & 2.97 & 0.24 & $<0.57$ & & 5939 & 4.35 & 1.09 & 1.13 & 0.11 & S & $\mathrm{N}$ & $\mathrm{Y}$ \\
\hline 10593535 & 20.925 & 67.900 & 0.93 & 0.10 & 3.63 & 0.59 & $<0.81$ & & 5822 & 4.28 & 1.25 & 1.27 & 0.13 & S & & $\mathrm{N}$ \\
\hline 10722485 & 7.849 & 67.907 & 0.87 & 0.09 & 2.61 & 0.51 & $<0.87$ & & 5682 & 4.36 & 1.03 & 0.98 & 0.10 & S & & $\mathrm{N}$ \\
\hline 10917433 & 6.912 & 65.190 & 0.51 & 0.05 & 2.03 & 0.57 & $<0.87$ & & 5680 & 4.33 & 1.12 & 0.62 & 0.06 & S & & $\mathrm{N}$ \\
\hline 11086270 & 31.720 & 75.821 & 1.90 & 0.19 & 3.35 & 0.40 & 0.70 & 0.14 & 5960 & 4.37 & 1.08 & 2.24 & 0.22 & $S$ & $\mathrm{~N}$ & $\mathrm{Y}$ \\
\hline 11121752 & 7.630 & 70.087 & 1.00 & 0.10 & 1.56 & 0.23 & $<0.75$ & & 6045 & 4.36 & 1.12 & 1.22 & 0.12 & S & $\mathrm{N}$ & $\mathrm{Y}$ \\
\hline 11133306 & 41.746 & 101.657 & 1.90 & 0.19 & 2.32 & 0.15 & 0.27 & 0.22 & 5953 & 4.37 & 1.10 & 2.27 & 0.23 & S & $\mathrm{N}$ & $\mathrm{Y}$ \\
\hline 11241912 & 14.427 & 71.857 & 0.95 & 0.10 & 2.40 & 0.18 & $<0.59$ & & 5931 & 4.40 & 1.07 & 1.10 & 0.11 & S & & $\mathrm{N}$ \\
\hline 11250587 & 7.257 & 67.028 & 1.95 & 0.20 & 2.41 & 0.05 & $<0.40$ & & 5853 & 4.18 & 1.49 & 3.18 & 0.32 & $\mathrm{~S}$ & $\mathrm{~N}$ & $\mathrm{Y}$ \\
\hline 11253711 & 17.791 & 82.259 & 1.86 & 0.65 & 1.29 & 0.09 & $<0.57$ & & 5816 & 4.48 & 0.95 & 1.92 & 0.67 & $\mathrm{P} 1$ & $\mathrm{~N}$ & $\mathrm{Y}$ \\
\hline 11295426 & 5.399 & 69.065 & 1.89 & 0.19 & 2.78 & 0.20 & 0.80 & 0.05 & 5793 & 4.25 & 1.30 & 2.68 & 0.27 & $\mathrm{~S}$ & $\mathrm{~N}$ & $\mathrm{Y}$ \\
\hline 11402995 & 10.061 & 71.959 & 2.00 & 0.20 & 2.42 & 0.19 & $<0.62$ & & 5709 & 4.30 & 1.18 & 2.56 & 0.26 & S & $\mathrm{N}$ & $\mathrm{Y}$ \\
\hline 11554435 & 9.434 & 73.119 & 5.69 & 0.57 & 1.29 & 0.02 & $<0.43$ & & 5536 & 4.52 & 0.90 & 5.60 & 0.56 & S & $\mathrm{N}$ & $\mathrm{Y}$ \\
\hline 11560897 & 35.968 & 71.667 & 1.46 & 0.15 & 1.47 & 0.11 & $<0.45$ & & 5832 & 4.27 & 1.28 & 2.03 & 0.21 & S & $\mathrm{N}$ & $\mathrm{Y}$ \\
\hline 11612280 & 9.406 & 70.699 & 0.80 & 0.08 & 3.02 & 0.54 & $<0.77$ & & 5857 & 4.24 & 1.32 & 1.15 & 0.12 & $\mathrm{~S}$ & & $\mathrm{~N}$ \\
\hline 11771430 & 40.031 & 81.863 & 1.39 & 0.14 & 2.25 & 0.14 & $<0.57$ & & 5850 & 4.23 & 1.38 & 2.10 & 0.21 & $\mathrm{~S}$ & $\mathrm{~N}$ & $\mathrm{Y}$ \\
\hline 11774991 & 37.815 & 74.112 & 1.45 & 0.15 & 2.37 & 0.21 & $<0.65$ & & 4710 & 4.62 & 0.70 & 1.10 & 0.11 & $\mathrm{~S}$ & $\mathrm{~N}$ & $\mathrm{Y}$ \\
\hline 12254909 & 5.350 & 66.872 & 0.84 & 0.29 & 2.26 & 0.14 & $<0.60$ & & 5987 & 4.46 & 0.96 & 0.88 & 0.31 & $\mathrm{P} 1$ & $\mathrm{~N}$ & $\mathrm{Y}$ \\
\hline 12301181 & 6.147 & 67.867 & 1.04 & 0.11 & 1.45 & 0.07 & $<0.40$ & & 4997 & 4.60 & 0.74 & 0.84 & 0.09 & $\mathrm{~S}$ & $\mathrm{~N}$ & $\mathrm{Y}$ \\
\hline 12416661 & 8.053 & 67.968 & 0.63 & 0.22 & 2.93 & 0.37 & $<0.59$ & & 6091 & 4.12 & 1.47 & 1.02 & 0.36 & $\mathrm{P} 1$ & & $\mathrm{~N}$ \\
\hline 12454461 & 7.467 & 69.392 & 0.84 & 0.09 & 1.83 & 0.20 & $<0.71$ & & 6048 & 4.31 & 1.27 & 1.16 & 0.12 & $\mathrm{~S}$ & $\mathrm{~N}$ & $\mathrm{Y}$ \\
\hline 12737015 & 24.669 & 69.616 & 1.05 & 0.11 & 4.94 & 0.49 & $<0.69$ & & 6045 & 4.15 & 1.60 & 1.84 & 0.19 & S & & $\mathrm{N}$ \\
\hline
\end{tabular}

Notes. Orbital period, $P$; time of transit center, $t_{0}$; planet-to-star radius ratio, $R_{P} / R_{\star}$; the time for the planet to travel $R_{\star}$ during transit, $\tau$; and transit impact parameter, $b$ are all determined from the Mandel \& Agol (2002) light curve fit. By default, stellar parameters $R_{\star}, T_{\text {eff }}$, and $\log g$ come from SpecMatch. If SpecMatch parameters do not exist, parameters are taken from the corrected KIC values, described in Section 4. The FP column lists whether a candidate was designated a false positive by the Kepler team ("Y"—yes, "N"—no, blank—no designation). The B12 column lists whether a candidate was present in Batalha et al. (2012).

${ }^{\text {a }}$ Time of transit center (BJD-2454900).

b Source of stellar parameters: "S"-SpecMatch-derived parameters using Keck HIRES spectra, "P1"- photometrically-derived parameters from Batalha et al. (2012), "P2"- photometrically-derived parameters computed by the authors. See Section 4 for more details.

Table 3

Union of Batalha et al. (2012) and TERRA Planet Candidate Catalogs

\begin{tabular}{lrrrrrr}
\hline \hline KIC & \multicolumn{3}{c}{ Batalha } & & \multicolumn{2}{c}{ TERRA } \\
\cline { 2 - 3 } \cline { 5 - 6 } & \multicolumn{1}{c}{ KOI } & $P$ & $R_{P}$ & & \multicolumn{1}{c}{$R_{P}$} \\
\hline 2142522 & & & & 13.32 & 1.11 \\
2307415 & 2053.01 & 13.12 & 1.65 & 13.12 & 1.57 \\
2441495 & 166.01 & 12.49 & 2.70 & 12.49 & 1.99 \\
2444412 & 103.01 & 14.91 & 2.97 & 14.91 & 3.41 \\
2571238 & 84.01 & 9.29 & 2.53 & 9.29 & 2.82 \\
2853446 & 118.01 & 7.37 & 2.52 & 7.37 & 1.65 \\
3098810 & 1878.01 & 40.81 & 3.24 & 40.81 & 2.75 \\
3120904 & & & & 42.91 & 1.59 \\
3342794 & 2278.01 & 14.17 & 1.99 & 14.17 & 1.68 \\
3442055 & 1218.01 & 29.62 & 2.22 & 29.62 & 1.72 \\
3531558 & 118.01 & 24.99 & 1.41 & & 24.99 & 1.85 \\
3545135 & & & & 8.48 & 0.90 \\
3835670 & 149.01 & 14.56 & 5.50 & 14.56 & 4.93
\end{tabular}


Table 3

(Continued)

\begin{tabular}{|c|c|c|c|c|c|}
\hline \multirow[t]{2}{*}{ KIC } & \multicolumn{3}{|c|}{ Batalha } & \multicolumn{2}{|c|}{ TERRA } \\
\hline & KOI & $P$ & $R_{P}$ & $P$ & $R_{P}$ \\
\hline 3839488 & 1216.01 & 11.13 & 1.57 & 11.13 & 1.64 \\
\hline 3852655 & & & & 11.63 & 0.97 \\
\hline 3942670 & 392.02 & 12.61 & 1.33 & & \\
\hline 3942670 & 392.01 & 33.42 & 2.27 & 33.42 & 2.13 \\
\hline 4043190 & 1220.01 & 6.40 & 1.95 & 6.40 & 2.86 \\
\hline 4049901 & 2295.01 & 16.29 & 0.63 & 16.29 & 0.60 \\
\hline 4548011 & & & & 6.28 & 0.83 \\
\hline 4644604 & 628.01 & 14.49 & 1.87 & 14.49 & 2.32 \\
\hline 4770174 & & & & 6.10 & 0.63 \\
\hline 4827723 & 632.01 & 7.24 & 1.46 & 7.24 & 1.54 \\
\hline 4914423 & 108.01 & 15.97 & 2.94 & 15.97 & 3.05 \\
\hline 4914566 & & & & 22.24 & 1.18 \\
\hline 5009743 & 1609.01 & 41.70 & 2.34 & 41.70 & 2.32 \\
\hline 5042210 & 2462.01 & 12.15 & 1.37 & 12.15 & 1.16 \\
\hline 5094751 & 123.01 & 6.48 & 2.64 & 6.48 & 2.02 \\
\hline 5094751 & 123.02 & 21.22 & 2.71 & & \\
\hline 5096590 & & & & 29.61 & 0.79 \\
\hline 5121511 & 640.01 & 31.00 & 2.43 & 31.00 & 2.15 \\
\hline 5308537 & & & & 14.27 & 1.05 \\
\hline 5446285 & 142.01 & 10.92 & 4.08 & & \\
\hline 5561278 & 1621.01 & 20.31 & 2.48 & 20.31 & 1.45 \\
\hline 5613330 & 649.01 & 23.45 & 2.31 & 23.45 & 2.70 \\
\hline 5652893 & & & & 14.01 & 0.97 \\
\hline 5702939 & & & & 18.40 & 1.04 \\
\hline 5735762 & 148.02 & 9.67 & 3.14 & 9.67 & 2.51 \\
\hline 5735762 & 148.03 & 42.90 & 2.35 & & \\
\hline 5866724 & 85.01 & 5.86 & 2.35 & 5.86 & 2.37 \\
\hline 5866724 & 85.03 & 8.13 & 1.41 & & \\
\hline 5959719 & 2498.01 & 6.74 & 0.78 & 6.74 & 0.83 \\
\hline 6061773 & 2001.01 & 8.28 & 2.39 & & \\
\hline 6071903 & 306.01 & 24.31 & 2.28 & 24.31 & 2.06 \\
\hline 6197215 & & & & 10.61 & 1.46 \\
\hline 6289257 & 307.02 & 5.21 & 1.15 & & \\
\hline 6289257 & 307.01 & 19.67 & 1.80 & 19.67 & 1.61 \\
\hline 6291837 & 308.01 & 35.60 & 3.15 & 35.60 & 3.05 \\
\hline 6356692 & & & & 11.39 & 1.19 \\
\hline 6365156 & 662.01 & 10.21 & 2.05 & 10.21 & 2.13 \\
\hline 6442340 & 664.02 & 7.78 & 1.19 & & \\
\hline 6442340 & 664.01 & 13.14 & 1.83 & 13.14 & 1.63 \\
\hline 6442340 & 664.03 & 23.44 & 1.19 & & \\
\hline 6521045 & 41.02 & 6.89 & 1.23 & & \\
\hline 6521045 & 41.01 & 12.82 & 2.08 & 12.82 & 1.85 \\
\hline 6521045 & 41.03 & 35.33 & 1.40 & & \\
\hline 6523351 & & & & 6.07 & 1.06 \\
\hline 6605493 & 2559.01 & 9.31 & 0.99 & 9.31 & 1.20 \\
\hline 6607357 & & & & 7.70 & 0.84 \\
\hline 6678383 & 111.01 & 11.43 & 2.14 & & \\
\hline 6678383 & 111.02 & 23.67 & 2.05 & & \\
\hline 6707835 & 666.01 & 22.25 & 2.56 & 22.25 & 2.42 \\
\hline 6716545 & & & & 13.91 & 1.03 \\
\hline 6803202 & 177.01 & 21.06 & 1.84 & 21.06 & 1.77 \\
\hline 6850504 & 70.04 & 6.10 & 0.91 & & \\
\hline 6850504 & 70.01 & 10.85 & 3.09 & & \\
\hline 6850504 & 70.05 & 19.58 & 1.02 & & \\
\hline 6851425 & 163.01 & 11.12 & 2.27 & 11.12 & 1.80 \\
\hline 6922710 & 2087.01 & 23.13 & 1.54 & 23.13 & 1.18 \\
\hline 7021534 & & & & 9.07 & 1.35 \\
\hline 7033671 & 670.01 & 9.49 & 1.92 & 9.49 & 2.03 \\
\hline 7211221 & 1379.01 & 5.62 & 1.06 & 5.62 & 1.23 \\
\hline 7219825 & 238.01 & 17.23 & 2.40 & 17.23 & 2.54 \\
\hline 7219825 & 238.02 & 26.69 & 1.35 & & \\
\hline 7345248 & & & & 5.66 & 0.81 \\
\hline 7419318 & 313.02 & 8.44 & 1.61 & & \\
\hline 7419318 & 313.01 & 18.74 & 2.20 & 18.74 & 1.89 \\
\hline 7466863 & & & & 11.97 & 2.89 \\
\hline
\end{tabular}


Table 3

(Continued)

\begin{tabular}{|c|c|c|c|c|c|}
\hline \multirow[t]{2}{*}{ KIC } & \multicolumn{3}{|c|}{ Batalha } & \multicolumn{2}{|c|}{ TERRA } \\
\hline & KOI & $P$ & $R_{P}$ & $P$ & $R_{P}$ \\
\hline 7582689 & & & & 11.92 & 1.34 \\
\hline 7668663 & 1898.01 & 6.50 & 1.50 & 6.50 & 1.79 \\
\hline 7700622 & 315.01 & 35.59 & 2.14 & 35.59 & 2.13 \\
\hline 7762723 & & & & 9.89 & 0.64 \\
\hline 7810483 & & & & 29.92 & 1.70 \\
\hline 7906739 & & & & 7.01 & 0.80 \\
\hline 7906892 & & & & 8.85 & 0.72 \\
\hline 7918652 & & & & 11.46 & 1.02 \\
\hline 8008067 & 316.01 & 15.77 & 2.72 & 15.77 & 2.66 \\
\hline 8009496 & & & & 38.48 & 1.74 \\
\hline 8073705 & & & & 10.60 & 0.91 \\
\hline 8077137 & 274.01 & 15.09 & 1.12 & 15.09 & 0.99 \\
\hline 8077137 & 274.02 & 22.80 & 1.13 & & \\
\hline 8081187 & & & & 37.32 & 1.57 \\
\hline 8087812 & & & & 27.21 & 1.55 \\
\hline 8242434 & 1726.01 & 44.96 & 5.25 & 44.96 & 1.92 \\
\hline 8280511 & 1151.01 & 5.22 & 0.84 & & \\
\hline 8280511 & 1151.02 & 7.41 & 0.97 & & \\
\hline 8280511 & & & & 10.44 & 1.36 \\
\hline 8323753 & & & & 6.71 & 2.71 \\
\hline 8349582 & 122.01 & 11.52 & 2.78 & 11.52 & 3.15 \\
\hline 8429668 & & & & 5.01 & 0.52 \\
\hline 8480285 & 691.02 & 16.23 & 1.25 & & \\
\hline 8480285 & 691.01 & 29.67 & 2.92 & 29.67 & 2.89 \\
\hline 8494617 & 2389.01 & 22.92 & 1.45 & 22.92 & 1.27 \\
\hline 8554498 & 5.02 & 7.05 & 0.66 & & \\
\hline 8560804 & & & & 31.98 & 1.09 \\
\hline 8611832 & 2414.01 & 22.60 & 1.03 & 22.60 & 1.17 \\
\hline 8611832 & 2414.02 & 45.35 & 1.17 & & \\
\hline 8628758 & 1279.02 & 9.65 & 0.74 & & \\
\hline 8628758 & 1279.01 & 14.37 & 1.31 & 14.37 & 2.26 \\
\hline 8631504 & 2503.01 & 14.82 & 2.41 & 14.82 & 0.95 \\
\hline 8644365 & & & & 19.92 & 1.31 \\
\hline 8804455 & 2159.01 & 7.60 & 1.01 & 7.60 & 1.33 \\
\hline 8805348 & 695.01 & 29.91 & 2.51 & 29.91 & 2.69 \\
\hline 8822366 & 1282.01 & 30.86 & 3.00 & 30.86 & 1.76 \\
\hline 8827575 & & & & 10.13 & 0.82 \\
\hline 8866102 & 42.01 & 17.83 & 2.71 & 17.83 & 2.08 \\
\hline 8962094 & 700.02 & 9.36 & 1.29 & & \\
\hline 8962094 & 700.03 & 14.67 & 1.29 & & \\
\hline 8962094 & 700.01 & 30.86 & 2.28 & 30.87 & 2.38 \\
\hline 8972058 & 159.01 & 8.99 & 2.70 & 8.99 & 2.39 \\
\hline 9006186 & 2169.01 & 5.45 & 1.02 & 5.45 & 0.81 \\
\hline 9086251 & 2367.01 & 6.89 & 1.17 & 6.89 & 1.38 \\
\hline 9139084 & 323.01 & 5.84 & 2.17 & 5.84 & 1.92 \\
\hline 9226339 & & & & 21.46 & 1.50 \\
\hline 9288237 & & & & 7.49 & 0.54 \\
\hline 9471974 & 119.01 & 49.18 & 3.76 & & \\
\hline 9491832 & & & & 49.57 & 1.95 \\
\hline 9549648 & 1886.01 & 5.99 & 2.45 & 5.99 & 1.82 \\
\hline 9704384 & 1913.01 & 5.51 & 1.40 & 5.51 & 1.35 \\
\hline 9716028 & & & & 17.37 & 1.03 \\
\hline 9717943 & 2273.01 & 6.11 & 1.02 & 6.11 & 1.00 \\
\hline 9886361 & & & & 7.03 & 1.08 \\
\hline 10055126 & 1608.01 & 9.18 & 1.81 & 9.18 & 1.59 \\
\hline 10055126 & 1608.02 & 19.74 & 1.58 & & \\
\hline 10130039 & 1909.02 & 5.47 & 1.15 & & \\
\hline 10130039 & 1909.01 & 12.76 & 1.52 & 12.76 & 1.31 \\
\hline 10130039 & 1909.03 & 25.10 & 1.63 & & \\
\hline 10136549 & 1929.01 & 9.69 & 2.00 & 9.69 & 1.98 \\
\hline 10212441 & 2342.01 & 15.04 & 1.22 & 15.04 & 1.13 \\
\hline 10593535 & & & & 20.92 & 1.27 \\
\hline 10722485 & & & & 7.85 & 0.98 \\
\hline 10917433 & & & & 6.91 & 0.62 \\
\hline 11086270 & 124.01 & 12.69 & 3.00 & & \\
\hline
\end{tabular}


Table 3

(Continued)

\begin{tabular}{lrrrrr}
\hline \hline KIC & \multicolumn{2}{c}{ Batalha } & & \multicolumn{2}{c}{ TERRA } \\
\cline { 2 - 4 } & \multicolumn{1}{c}{ KOI } & \multicolumn{1}{c}{$P$} & $R_{P}$ & & \multicolumn{1}{c}{$P$} \\
\hline 11086270 & 124.02 & 31.72 & 3.58 & 31.72 & $R_{P}$ \\
11121752 & 2333.02 & 7.63 & 1.63 & 7.63 & 1.22 \\
11133306 & 276.01 & 41.75 & 2.49 & 41.75 & 2.27 \\
11241912 & & & & 14.43 & 1.10 \\
11250587 & 107.01 & 7.26 & 3.09 & 7.26 & 3.18 \\
11253711 & 1972.01 & 17.79 & 1.93 & 17.79 & 1.92 \\
11295426 & 246.01 & 5.40 & 2.53 & 5.40 & 2.68 \\
11402995 & 173.01 & 10.06 & 2.48 & 10.06 & 2.56 \\
11554435 & 63.01 & 9.43 & 6.30 & 9.43 & 5.60 \\
11560897 & 2365.01 & 35.97 & 1.59 & 35.97 & 2.03 \\
11612280 & & & & 9.41 & 1.15 \\
11771430 & 2582.01 & 40.03 & 1.98 & 40.03 & 2.10 \\
11774991 & 2173.01 & 37.82 & 1.24 & 37.82 & 1.10 \\
11818872 & 2581.01 & 12.74 & 0.90 & & \\
12254909 & 2372.01 & 5.35 & 1.11 & 5.35 & 0.88 \\
12301181 & 2059.01 & 6.15 & 0.59 & 6.15 & 0.84 \\
12416661 & & & & 8.05 & 1.02 \\
12454461 & 2463.01 & 7.47 & 1.07 & 7.47 & 1.16 \\
12737015 & & & & 24.67 & 1.84 \\
\hline
\end{tabular}

Notes. All Batalha et al. (2012) candidates with $P=5-50$ days belonging to stars in the Best12k sample are included. Candidates are considered equal if they belong to the same star and the periods in each catalog agree to better than 0.01 days. Eighty-two candidates appear in both catalogs, 33 appear in Batalha et al. (2012) only, and 47 appear in this work only (although 10 were listed as false positives by the Kepler team). Differences in $R_{P}$ between the two catalogs stem from different values of $R_{\star}$. Most TERRA planet candidates have SpecMatch-derived stellar parameters which are more accurate than Batalha et al. (2012) parameters, which were derived from KIC broadband photometry.

Table 4

New Candidates Identified with TERRA

\begin{tabular}{|c|c|c|c|c|c|c|c|c|c|c|c|c|c|c|}
\hline \multirow[b]{2}{*}{$\mathrm{KIC}$} & \multicolumn{8}{|c|}{ Light Curve Fit } & \multicolumn{3}{|c|}{ Stellar Parameters } & \multirow[b]{2}{*}{$\begin{array}{c}R_{P} \\
\left(R_{E}\right) \\
\end{array}$} & \multirow[b]{2}{*}{$\sigma\left(R_{P}\right)$} & \multirow[b]{2}{*}{ Source $^{b}$} \\
\hline & $\begin{array}{c}P \\
\text { (days) }\end{array}$ & $\begin{array}{c}t_{0}{ }^{\mathrm{a}} \\
\text { (days) }\end{array}$ & $\begin{array}{l}\frac{R_{P}}{R_{\star}} \\
(\%)\end{array}$ & $\sigma\left(\frac{R_{P}}{R_{\star}}\right)$ & $\begin{array}{c}\tau \\
(\mathrm{hr})\end{array}$ & $\sigma(\tau)$ & $b$ & $\sigma(b)$ & $\begin{array}{l}T_{\text {eff }} \\
(\mathrm{K})\end{array}$ & $\begin{array}{l}\log g \\
(\operatorname{cgs})\end{array}$ & $\begin{array}{c}R_{\star} \\
\left(R_{\odot}\right)\end{array}$ & & & \\
\hline 3120904 & 42.915 & 72.866 & 1.15 & 0.12 & 2.96 & 0.36 & $<0.66$ & & 6151 & 4.31 & 1.26 & 1.59 & 0.16 & $\mathrm{~S}$ \\
\hline 3545135 & 8.483 & 65.973 & 0.81 & 0.08 & 1.44 & 0.10 & $<0.57$ & & 5794 & 4.40 & 1.02 & 0.90 & 0.09 & $\mathrm{~S}$ \\
\hline 3852655 & 11.629 & 65.817 & 0.85 & 0.30 & 1.49 & 0.23 & $<0.79$ & & 5822 & 4.36 & 1.05 & 0.97 & 0.34 & P1 \\
\hline 4548011 & 6.284 & 66.198 & 0.60 & 0.06 & 2.23 & 0.64 & $<0.49$ & & 5991 & 4.30 & 1.26 & 0.83 & 0.09 & $\mathrm{~S}$ \\
\hline 4770174 & 6.096 & 67.600 & 0.57 & 0.20 & 3.08 & 0.44 & $<0.75$ & & 6013 & 4.44 & 1.01 & 0.63 & 0.22 & $\mathrm{P} 1$ \\
\hline 5096590 & 29.610 & 70.332 & 1.00 & 0.35 & 2.60 & 0.20 & $<0.67$ & & 5623 & 4.63 & 0.73 & 0.79 & 0.28 & $\mathrm{P} 1$ \\
\hline 5308537 & 14.265 & 76.217 & 0.76 & 0.08 & 1.92 & 0.53 & $<0.87$ & & 5831 & 4.29 & 1.26 & 1.05 & 0.11 & $\mathrm{~S}$ \\
\hline 5652893 & 14.010 & 65.959 & 1.15 & 0.13 & 1.98 & 0.49 & $<0.85$ & & 5150 & 4.55 & 0.78 & 0.97 & 0.11 & S \\
\hline 5702939 & 18.398 & 77.934 & 1.09 & 0.38 & 2.45 & 0.27 & $<0.54$ & & 5634 & 4.47 & 0.87 & 1.04 & 0.36 & $\mathrm{P} 1$ \\
\hline 6197215 & 10.613 & 68.691 & 1.23 & 0.13 & 0.48 & 0.06 & $<0.80$ & & 5933 & 4.39 & 1.09 & 1.46 & 0.15 & $\mathrm{~S}$ \\
\hline 6356692 & 11.392 & 74.555 & 0.66 & 0.23 & 2.74 & 0.31 & $<0.73$ & & 5420 & 4.03 & 1.64 & 1.19 & 0.42 & P1 \\
\hline 6523351 & 6.067 & 69.128 & 0.72 & 0.25 & 1.02 & 0.20 & $<0.89$ & & 5489 & 4.15 & 1.35 & 1.06 & 0.37 & P1 \\
\hline 6607357 & 7.700 & 67.390 & 0.85 & 0.30 & 2.72 & 0.45 & 0.55 & 0.22 & 5592 & 4.51 & 0.91 & 0.84 & 0.29 & $\mathrm{P} 1$ \\
\hline 6716545 & 13.910 & 75.855 & 0.84 & 0.30 & 3.29 & 0.68 & $<0.85$ & & 6044 & 4.30 & 1.12 & 1.03 & 0.36 & P1 \\
\hline 7345248 & 5.665 & 69.338 & 0.63 & 0.22 & 2.81 & 0.43 & $<0.61$ & & 5656 & 4.27 & 1.19 & 0.81 & 0.29 & $\mathrm{P} 2$ \\
\hline 7582689 & 11.921 & 70.475 & 0.75 & 0.26 & 5.84 & 1.24 & $<0.95$ & & 6022 & 4.04 & 1.64 & 1.34 & 0.47 & P1 \\
\hline 7906892 & 8.849 & 72.797 & 0.58 & 0.07 & 8.03 & 3.69 & 0.89 & 0.12 & 6095 & 4.35 & 1.14 & 0.72 & 0.08 & $\mathrm{~S}$ \\
\hline 7918652 & 11.456 & 69.316 & 0.79 & 0.28 & 2.45 & 0.47 & $<0.81$ & & 5809 & 4.25 & 1.19 & 1.02 & 0.36 & P1 \\
\hline 8073705 & 10.601 & 65.967 & 0.75 & 0.08 & 2.29 & 0.36 & $<0.80$ & & 6086 & 4.36 & 1.12 & 0.91 & 0.10 & $\mathrm{~S}$ \\
\hline 8087812 & 27.211 & 65.360 & 1.01 & 0.10 & 5.01 & 0.57 & $<0.67$ & & 5985 & 4.17 & 1.41 & 1.55 & 0.16 & S \\
\hline 8280511 & 10.435 & 67.826 & 1.37 & 0.14 & 1.70 & 0.12 & $<0.58$ & & 5522 & 4.45 & 0.91 & 1.36 & 0.14 & S \\
\hline 8429668 & 5.007 & 67.696 & 0.73 & 0.26 & 1.66 & 0.47 & 0.62 & 0.29 & 5034 & 4.63 & 0.65 & 0.52 & 0.18 & $\mathrm{P} 2$ \\
\hline 8560804 & 31.976 & 66.803 & 0.99 & 0.35 & 4.96 & 0.48 & $<0.65$ & & 5878 & 4.44 & 1.01 & 1.09 & 0.38 & $\mathrm{P} 1$ \\
\hline 8644365 & 19.917 & 72.354 & 1.07 & 0.11 & 3.37 & 0.55 & $<0.85$ & & 6054 & 4.39 & 1.12 & 1.31 & 0.13 & $\mathrm{~S}$ \\
\hline 8827575 & 10.129 & 68.997 & 0.84 & 0.30 & 1.94 & 0.18 & $<0.63$ & & 5284 & 4.45 & 0.89 & 0.82 & 0.29 & P1 \\
\hline 9226339 & 21.461 & 65.230 & 1.10 & 0.11 & 1.66 & 0.16 & $<0.70$ & & 5807 & 4.28 & 1.25 & 1.50 & 0.15 & $\mathrm{~S}$ \\
\hline 9288237 & 7.491 & 68.329 & 0.52 & 0.18 & 3.01 & 0.73 & $<0.84$ & & 5946 & 4.44 & 0.96 & 0.54 & 0.19 & $\mathrm{P} 1$ \\
\hline 9491832 & 49.565 & 103.693 & 1.14 & 0.12 & 6.43 & 1.49 & 0.74 & 0.25 & 5821 & 4.15 & 1.57 & 1.95 & 0.21 & $S$ \\
\hline 9716028 & 17.373 & 71.258 & 0.82 & 0.08 & 2.46 & 0.27 & $<0.73$ & & 6119 & 4.37 & 1.15 & 1.03 & 0.11 & $S$ \\
\hline 9886361 & 7.031 & 67.464 & 0.88 & 0.09 & 3.04 & 0.21 & $<0.42$ & & 6090 & 4.39 & 1.13 & 1.08 & 0.11 & $\mathrm{~S}$ \\
\hline 10593535 & 20.925 & 67.900 & 0.93 & 0.10 & 3.63 & 0.59 & $<0.81$ & & 5822 & 4.28 & 1.25 & 1.27 & 0.13 & S \\
\hline
\end{tabular}


Table 4

(Continued)

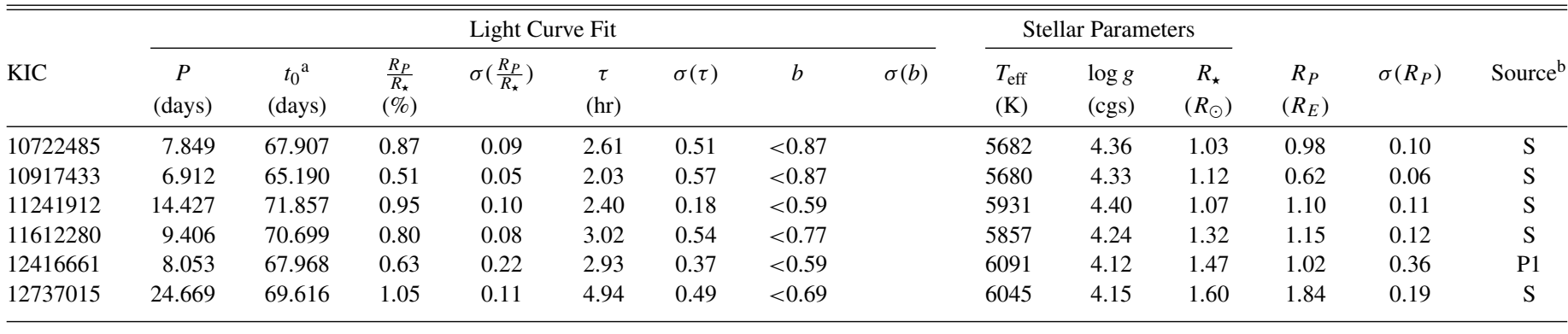

Notes. The 37 TERRA candidates not in Batalha et al. (2012) and not listed as false positives by the Kepler team. The column definitions are the same as in Table 2 .

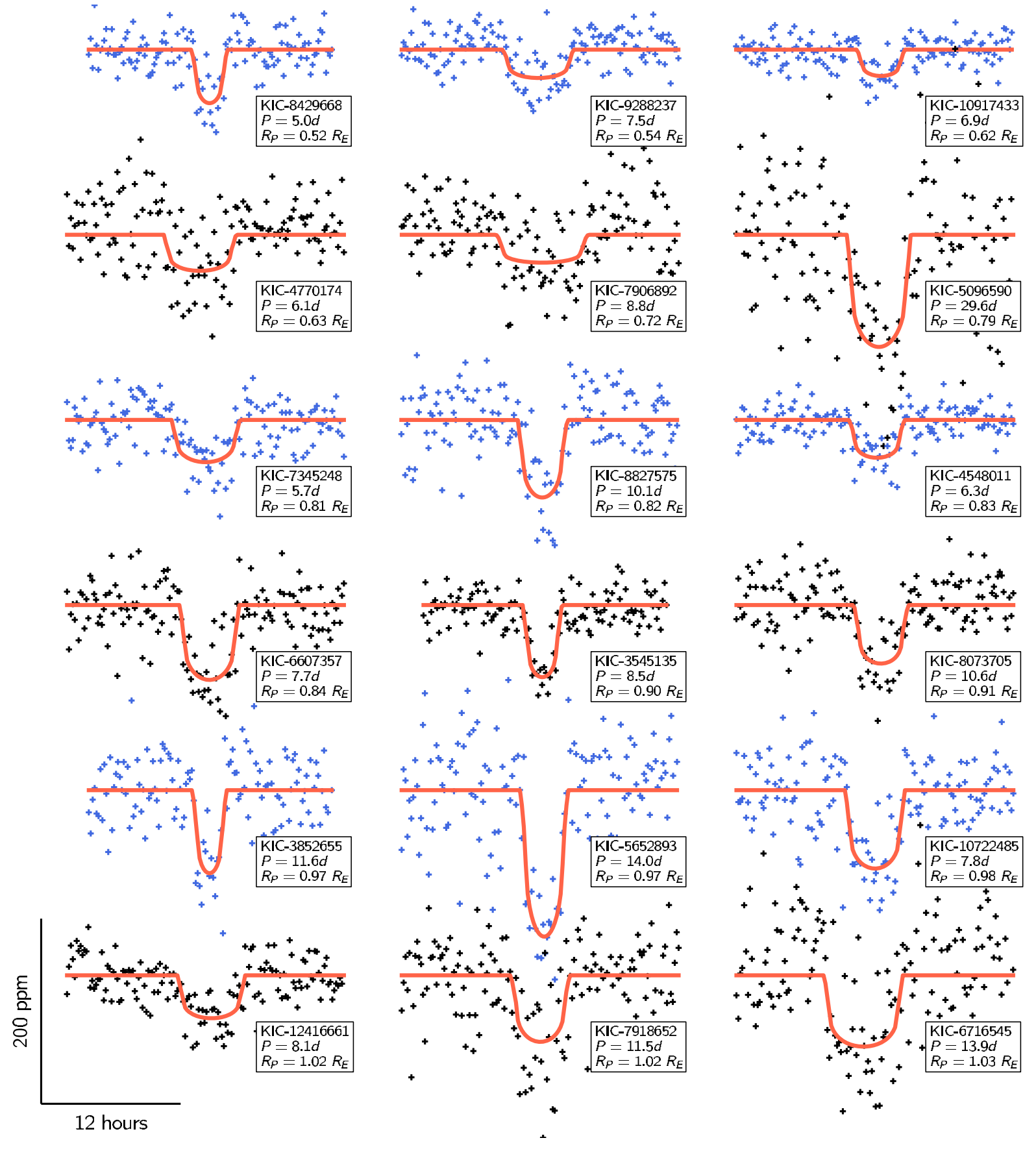

Figure 17. Phase-folded photometry for 18 of the 37 TERRA planet candidates, not in Batalha et al. (2012), ordered according to size. For clarity, we show median photometric measurements in 10 minute bins. The red lines are the best-fitting Mandel \& Agol (2002) model.

(A color version of this figure is available in the online journal.) 

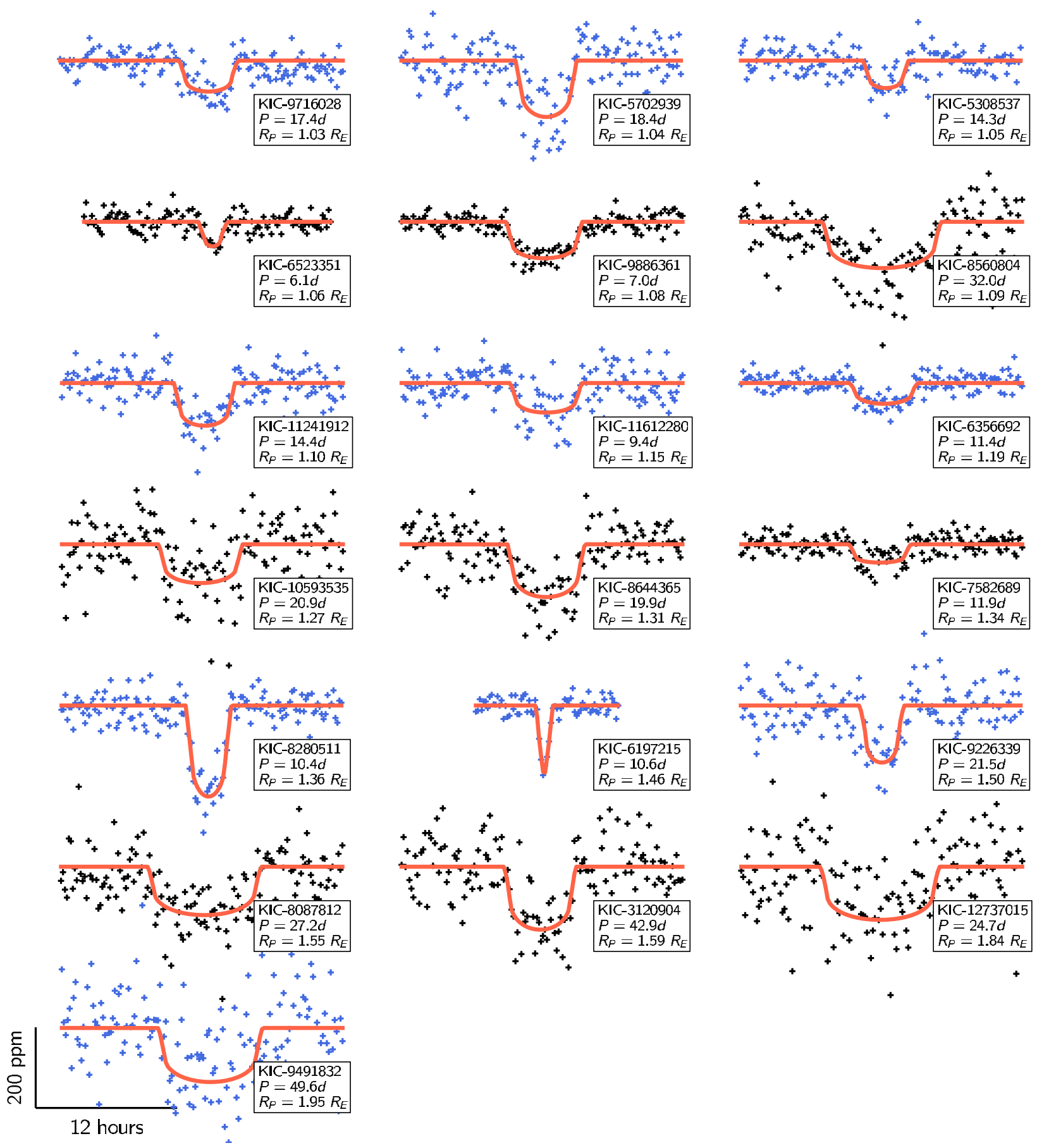

Figure 18. Same as Figure 17, but showing the remaining 19 of the 37 TERRA planet candidates, not in Batalha et al. (2012).

(A color version of this figure is available in the online journal.)

\section{REFERENCES}

Adams, E. R., Seager, S., \& Elkins-Tanton, L. 2008, ApJ, 673, 1160 Batalha, N. M., Rowe, J. F., Bryson, S. T., et al. 2013, ApJS, 204, 24

Beaugé, C., \& Nesvorný, D. 2013, ApJ, 763, 12

Borucki, W. J., Koch, D. G., Basri, G., et al. 2011a, ApJ, 728, 117

Borucki, W. J., Koch, D. G., Basri, G., et al. 2011b, ApJ, 736, 19

Brown, T. M., Latham, D. W., Everett, M. E., \& Esquerdo, G. A. 2011, AJ, 142,112

Chiang, E., \& Laughlin, G. 2013, MNRAS, 431, 3444

Christiansen, J. L., Clarke, B. D., Burke, C. J., et al. 2013, arXiv:1303.0255

Collette, A. 2008, HDF5 for Python, http://h5py.alfven.org

Demarque, P., Woo, J.-H., Kim, Y.-C., \& Yi, S. K. 2004, ApJS, 155, 667
Dong, S., \& Zhu, Z. 2012, arXiv:1212.4853

Fang, J., \& Margot, J.-L. 2012, ApJ, 761, 92

Fraquelli, D., \& Thompson, S. E. 2012, Kepler Archive Manual (KDMC-10008004)

Fressin, F., Torres, G., Charbonneau, D., et al. 2013, ApJ, 766, 81

Hansen, B. M. S., \& Murray, N. 2012, ApJ, 751, 158

Howard, A. W., Marcy, G. W., Bryson, S. T., et al. 2012, ApJS, 201, 15

Howard, A. W., Marcy, G. W., Johnson, J. A., et al. 2010, Sci, 330, 653

Hunter, J. D. 2007, CSE, 9, 90

Ida, S., \& Lin, D. N. C. 2010, ApJ, 719, 810

Jenkins, J. M., Caldwell, D. A., Chandrasekaran, H., et al. 2010a, ApJL, 713, L87 
Jenkins, J. M., Chandrasekaran, H., McCauliff, S. D., et al. 2010b, Proc. SPIE, 7740, 77400D

Jones, E., Oliphant, T., Peterson, P., et al. 2001, SciPy: Open Source Scientific Tools for Python, www.scipy.org

Kovács, G., Zucker, S., \& Mazeh, T. 2002, A\&A, 391, 369

Lissauer, J. J., Ragozzine, D., Fabrycky, D. C., et al. 2011, ApJS, 197, 8

Mandel, K., \& Agol, E. 2002, ApJL, 580, L171

Marcy, G. W., Butler, R. P., Vogt, S. S., et al. 2008, PhST, 130, 014001

Mayor, M., Marmier, M., Lovis, C., et al. 2011, arXiv:1109.2497

Mordasini, C., Alibert, Y., \& Benz, W. 2009, A\&A, 501, 1139

Morton, T. D. 2012, ApJ, 761, 6

Morton, T. D., \& Johnson, J. A. 2011, ApJ, 738, 170

Muirhead, P. S., Johnson, J. A., Apps, K., et al. 2012, ApJ, 747, 144
North, D. O. 1963, Proc. IEEE, 51, 1016

Oliphant, T. E. 2007, CSE, 9, 10

Pérez, F., \& Granger, B. E. 2007, CSE, 9, 21

Petigura, E. A., \& Marcy, G. W. 2012, PASP, 124, 1073

Smith, J. C., Stumpe, M. C., Van Cleve, J. E., et al. 2012, PASP, 124, 1000

Staelin, D. H. 1969, IEEEP, 57, 724

Stumpe, M. C., Smith, J. C., Van Cleve, J. E., et al. 2012, PASP, 124, 985

Traub, W. A. 2012, ApJ, 745, 20

Twicken, J. D., Chandrasekaran, H., Jenkins, J. M., et al. 2010, Proc. SPIE, 7740, $77401 \mathrm{U}$

Vogt, S. S., Allen, S. L., Bigelow, B. C., et al. 1994, Proc. SPIE, 2198, 362

Youdin, A. N. 2011, ApJ, 742, 38 